\title{
Secondary Electrons and Photons in the Upper Atmosphere
}

\author{
Klaus P. Beuerman ${ }^{1}$ \\ California Institute of Technology, Pasadena, California 91109
}

\begin{abstract}
We present a theoretical and experimental study of the production and propagation of atmospheric secondary negatrons, positrons, and photons moving in the vertical direction. The one-dimensional transport equations have been solved numerically, to obtain energy spectra and intensity versus depth for energies as low as $4 \mathrm{Mev}$ and for atmospheric depths up to $400 \mathrm{~g} / \mathrm{cm}^{2}$. The calculations are performed for the solar modulation level of 1968 and geomagnetic cutoff values of zero and $4.5 \mathrm{Gv}$. We also present experimental data on negatrons and positrons in the energy range from 6.5 to $200 \mathrm{Mev}$ obtained from balloon observations near Ft. Churchill, Canada, in 1968. The comparisons with measurements confirm the calculated energy spectra and intensity versus depth. The spectral shapes of secondary negatrons and positrons are found to be very dependent upon the atmospheric depth. In the high atmosphere, the photon spectrum below $\sim 70 \mathrm{Mev}$ is dominated by bremsstrahlung from primary and reentrant albedo electrons rather than by $\pi^{0}$ decays. The derived intensity versus depth deviates distinctly from the commonly assumed linear growth of the secondary flux with increasing depth. Our results on the functional depth dependence are useful for determining the contribution from atmospheric secondaries to measurements of the electron (and photon) flux at balloon altitudes.
\end{abstract}

In recent years experiments to measure the flux and energy spectrum of cosmic ray electrons (i.e., negatrons and positrons) and photons have been conducted almost exclusively by means of high-altitude balloons. The residual atmosphere of $\gtrsim^{2} \mathrm{~g} / \mathrm{cm}^{2}$ typical for the float period is a source of secondary electrons and photons produced in interactions of primary cosmic rays with air nuclei. The minimum primary flux detectable from balloon altitudes is therefore limited by (1) the counting statistics and (2) the accuracy of our knowledge of the production and propagation of atmospheric secondaries.

At balloon altitudes, the flux of galactic cosmic ray electrons with energies above $\sim 1$ Gev has been shown to be significantly above the atmospheric background [e.g., Beedle and Webber, 1968; Bleeker et al., 1968; L'Heureux and Meyer, 1968]. At electron energies below a few hundred Mev, however, contamination by atmospheric secondary electrons represents a serious problem [Israel and Vogt, 1968; Israel, 1969; Beuermann et al., 1969b]. The flux of

\footnotetext{
${ }^{1}$ Now at Institut für Reine und Angewandte Kernphysik, 23 Kiel, Olshausenstr. 40-60, West Germany.
}

Copyright @ 1971 by the American Geophysical Union. galactic photons seems to be completely masked by the atmospheric background at all energies exceeding $\sim 1 \mathrm{Mev}$ [e.g., Peterson et al., 1967; Duthie, 1968]. Thus, a crucial problem in the interpretation of the low-energy electron (and photon) observations is the separation of primary particles incident on the top of the atmosphere and secondaries produced in the residual atmosphere above the detector. Usually, the separation of the two components is attempted by means of their distinctly different dependence on atmospheric depth. However, the functional depth dependence of the atmospheric flux is not known to great accuracy. Israel and Vogt [1968] pointed out that this uncertainty might significantly influence the conclusions on the primary flux. Thus, a clean measurement of the low extraterrestrial flux will not depend so much on improved experimental observations as on a better understanding of the production and propagation of the atmospheric component. Therefore, a more refined description of the build-up of the secondary intensity with atmospheric depth is needed.

Calculations of the production of secondaries in the upper atmosphere have been published by Perola and Scarsi [1966] and Verma [1967]. They utilized the known spectrum of primary 
cosmic ray nuclei at solar minimum and accelerator-determined pion production spectra to obtain the flux and energy spectra of secondary electrons between $10 \mathrm{Mev}$ and several Gev. Since the energy loss of electrons and the development of electromagnetic cascades were neglected, the authors quoted results only for the first few $\mathrm{g} / \mathrm{cm}^{2}$ of atmosphere. The propagation of secondary electrons and photons deep into the atmosphere has been considered by Okuda and Yamamoto [1965]. However, the analytical solutions of cascade theory utilized by these authors restrict the validity of their results to energies exceeding $\sim 1 \mathrm{Gev}$.

In this paper we present results of a numerical solution of the one-dimensional transport equations describing the development of the atmospheric secondary electron and photon component in the vertical direction. Extending the propagation study to energies as low as $4 \mathrm{Mev}$, we account for a number of factors not considered in previous calculations. These include (1) using the energy-dependent cross sections for bremsstrahlung, pair production, and the Compton effect, (2) accounting for energy loss of electrons by ionization and the production of knock-on negatrons, (3) including secondaries produced by primary and reentrant albedo electrons, (4) calculating the pion production rate as a function of atmospheric depth, and (5) accounting for the solar cycle modulation of cosmic rays.

We find that the calculations quite accurately reproduce the experimentally obtained energy spectra and the depth dependence of atmospheric secondaries. Remaining differences between observations and calculations are of a minor nature. The cascade development, the energy loss of electrons, and the production of secondaries by primary electrons are found to be important factors that must be included in a study of the production and propagation of lowenergy atmospheric electrons and photons.

\section{Production Spectra for 1968 at the top of THE ATMOSPHERE}

Most of the atmospheric secondary negatrons, positrons, and photons originate in the interactions of primary cosmic ray nuclei with air nuclei. In this and the following section, we derive the production spectra $Q_{t}(E, x)$ as a function of the energy $E$ and the atmospheric depth $x$, for particles moving in the vertical direction. (The suffix $i$ stands for the various particles, including pions). For the case of small atmospheric depths, $x \approx 0$, the production spectra have been calculated by Perola and Scarsi [1966] (personal communication by G. C. Perola) and by Verma [1967]. Their results, however, are not easily extrapolated to greater depths. Our approach will be (1) to use the absolute production spectra $Q_{i}(E, 0)$ at the top of the atmosphere, as given by Perola and Scarsi, and (2) to compute functions $D_{i}(E, x)$ that describe the attenuation of the production rates with atmospheric depth $x$, normalized to $D_{i}(E, 0)=1$. Thus

$$
\begin{array}{r}
Q_{i}(E, x)=Q_{i}(E, 0) \cdot D_{i}(E, x) \\
\text { particles/g sec ster Mev }
\end{array}
$$

The production spectra of Perola and Scarsi refer to times near solar minimum. They are based upon the fluxes and energy spectra of cosmic-ray protons and helium nuclei in 1965, as presented by Ormes and Webber [1966]. Given the cosmic ray spectra for a different level of solar modulation, the production spectra of Perola and Scarsi are easily adjusted for the change of the cosmic ray flux. We determine the production spectra for the time of our balloon flights in the summer of 1968 when the Mt. Washington neutron monitor level was 2200 (J. A. Lockwood, personal communication, 1968). We use the regression curves published by Webber [1967] to construct the integral kinetic energy/nucleon spectra of protons and helium nuclei for this modulation level. At high energies, we adjust the spectra to recent data on protons and helium nuclei above $10 \mathrm{Gev} /$ nucleon [Pinkau et al., 1969; Anand et al., 1968a]. The 1968 spectra can be described by the analytical expressions

$$
\begin{gathered}
J_{p}(E)=1.18 \times 10^{4}(E+3.3)^{-1.7} \\
\text { protons } / \mathrm{m}^{2} \text { sec ster } \\
J_{\alpha}(E)=4.65 \times 10^{2}(E+1.5)^{-1.6} \\
\text { helium nuclei } / \mathrm{m}^{2} \text { sec ster }
\end{gathered}
$$

with $E$ in Gev/nucleon. For multiply charged cosmic ray nuclei, we assume the same spectral shape and relative solar modulation as for helium nuclei. Using the $Z$ abundances given by 
Ormes and Webber [1966], Webber and Ormes [1967], and Mathiesen et al. [1968], we obtain a mean $Z^{2}$ equal to 11.8 for all $Z>1$ particles. The total flux of multiply charged cosmic ray nuclei is given by

$$
J_{Z>2}(E)=0.10 J_{\alpha}(E)
$$

(Simultaneously with our negatron-positron observations in summer 1968, we measured an integral flux of nuclei above approximately 450 $\mathrm{Mev} /$ nucleon of $1460 \pm 30 \mathrm{particles} / \mathrm{m}^{2}$ sec ster, which agrees with the flux of 1447 particles $/ \mathrm{m}^{2}$ sec ster derived from the above representations 2 to 4 ).

Figure 1 shows the Perola and Scarsi production spectra $Q_{e}(E, 0)$ of electrons from $\pi^{ \pm} \rightarrow$ $\mu^{ \pm} \rightarrow e^{ \pm}$decays and $Q_{\gamma}(E, 0)$ of photons from $\pi^{0} \rightarrow 2 \gamma$ decays adjusted for the difference in the cosmic ray flux between 1965 and 1968, as given by Ormes and Webber [1966] and by (2). Solid curves are for a geomagnetic cutoff $<0.5$ $\mathrm{Gv}$, dashed curves are for $4.5 \mathrm{Gv}$. For energies near the maximum of the spectra, the fluxes in 1965 and 1968 differ by a factor of 2.0 and 1.7 for geomagnetic cutoffs of $<0.5 \mathrm{Gv}$ and $4.5 \mathrm{Gv}$, respectively. Also shown in Figure 1 are the production spectra of knock-on negatrons for the same two values of the cutoff. These spectra are numerically calculated for a flux of cosmic ray protons and heavier nuclei as given by (2) to (4). Since the cross section for a collision of a charge $Z$ particle with an atomic electron [Rossi, 1952] is proportional to $Z^{2}$, multiply charged nuclei contribute strongly to this source. For zero geomagnetic cutoff, we find that $\sim 0.46$ of the knock-on negatrons at $10 \mathrm{Mev}$ are produced by particles heavier than protons (the fraction varying slightly with energy). In addition to knock-on negatrons produced by the nucleonic component, there will also be knockons created by electrons. This source will be discussed below in connection with the electromagnetic cascade process.

\section{Depth Dependence of the Production Rates}

We next discuss the function $D_{\mathfrak{i}}(E, x)$ in (1). For a pure power-law shape of the cosmic ray spectrum, the flux of high-energy nucleons is attenuated exponentially with atmospheric depth. The attenuation length $\Lambda$ is related to the nucleon interaction length $\lambda=80 \mathrm{~g} / \mathrm{cm}^{2}$ by [e.g., Brooke et al., 1964]

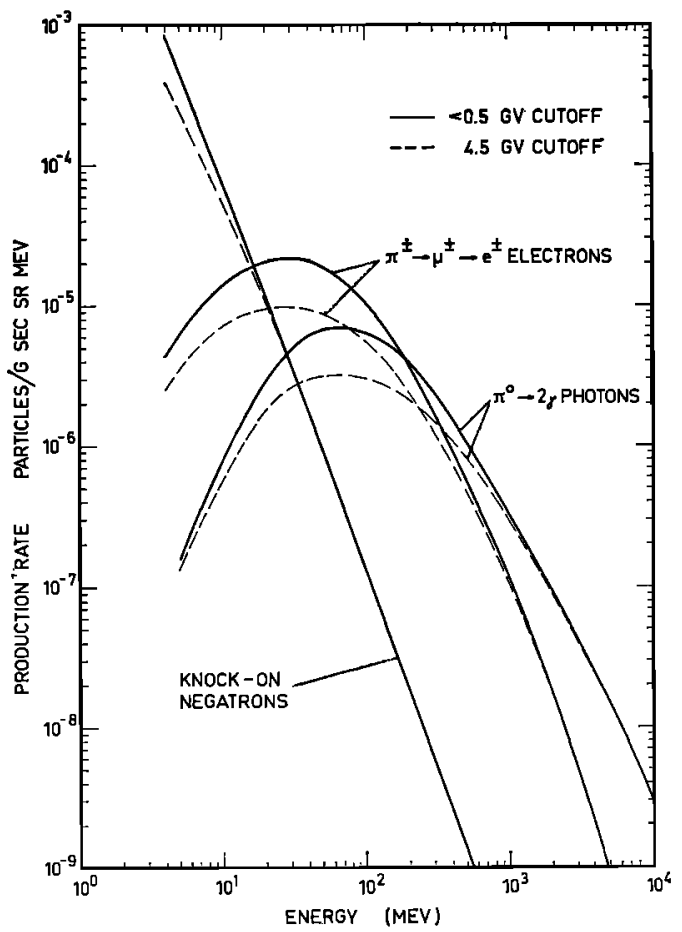

Fig. 1. Production spectra of downward-moving electrons and photons in 1968 for two different values of the geomagnetic cutoff. Except for the knock-on negatrons, the spectra are derived from the work of Perola and Scarsi [1966].

$$
\Lambda=\lambda /\left[1-(1-K)^{\gamma}\right]
$$

where $1-K \approx 0.5$ is the mean fractional energy retained by a colliding nucleon, and $\gamma=1.7$ is the power index of the cosmic ray spectrum. Equation 5 yields $\Lambda \approx 120 \mathrm{~g} / \mathrm{cm}^{2}$, in agreement with observations [Perkins, 1961]. The production rates of high-energy pions and of photons (as immediate decay products) are also attenuated exponentially with the same attenuation length $\Lambda$. The production rate of highenergy $\pi \rightarrow \mu \rightarrow e$ decay electrons has a corresponding attenuation length of approximately $\Lambda_{s}=2 \Lambda$ [Okuda and Yamamoto, 1965]. Thus, for secondaries with energies above a few Gev,

$$
\begin{aligned}
& D_{\mathbf{x}}\left(E_{\boldsymbol{x}}, x\right)=D_{\gamma}\left(E_{\gamma}, x\right)=\exp (-x / \Lambda) \\
& D_{\bullet}\left(E_{\bullet}, x\right)=\exp (-x / 2 \Lambda)
\end{aligned}
$$

with $\Lambda=120 \mathrm{~g} / \mathrm{cm}^{2}$. At lower energies, the cosmic ray spectrum loses the power-law shape and (5) is not applicable for determining the depth dependence of the nucleon flux. Observa- 
tions indicate pronounced deviations from an exponential attenuation [e.g., Lord, 1951; Chow et al., 1968]. Furthermore, the production of low-energy pions is characterized by a wide angular spread with respect to the trajectory of the incident nucleus. Thus the pion flux in the vertical direction will depend on the flux of nuclei traveling at different directions to the vertical. Our approach involves a numerical treatment of the propagation of nuclei through the atmosphere. We use a simple model of the nucleonic cascade to compute the energy spectra of protons and (secondary) neutrons as a function of energy, atmospheric depth, and zenith angle. The model is described in the Appendix. The production rate of pions moving in the vertical direction is then calculated numerically as a function of depth for pions produced by nucleons and by nuclei heavier than protons. Two special cases are considered: high-energy pions with energies above $\sim 10 \mathrm{Gev}$, and lowenergy pions with kinetic energy below $\sim 200$ Mev. For intermediate energies, we perform a suitable interpolation. At high energies, the use of the one-dimensional approximation is justified, and the solution can be compared with the observed exponential attenuation. The depth

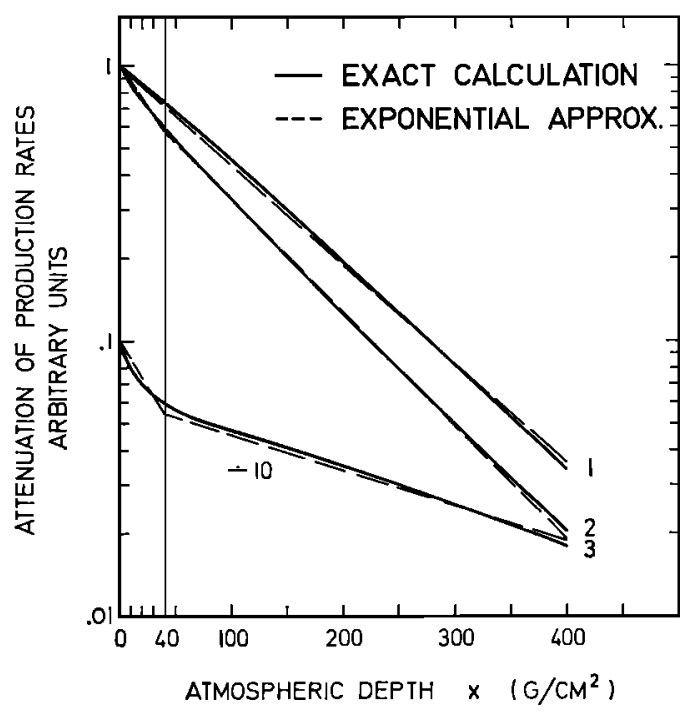

Fig. 2. Attenuation of the production rates with atmospheric depth for $<0.5-\mathrm{Gv}$ cutoff. Curve 1 is for pions above $\sim 10 \mathrm{Gev}$, curve 2 for pions below $\sim 200 \mathrm{Mev}$, and curve 3 is for knock-on negatrons. For the present calculations, we have used the exponential approximations indicated by dashed lines. dependence at low energies is obtained with the assumption of an isotropic production of pions below $\sim 200 \mathrm{Mev}$. Details of this calculation are also given in the Appendix.

In Figure 2, we show the resulting depth dependence $D_{\pi}\left(E_{\pi}, x\right)$ for the production rate of pions traveling vertically downward, calculated for 1968 and zero geomagnetic cutoff. Curve 1 represents the case of high energies and is well approximated by an exponential decay $\exp (-x / \Lambda)$ with $\Lambda=120 \mathrm{~g} / \mathrm{cm}^{2}$ (dashed line), as is expected from (5). Curve 2 is for pions with kinetic energy below $\sim 200 \mathrm{Mev}$. The rapid initial decay of the production rate is caused both by the contribution of multiply charged nuclei to pion production near the top of the atmosphere and by the change in the zenith angle distribution of the nucleonic component with increasing depth. It is clear from the difference between curve 1 and 2 that the shape of the production spectrum changes with depth, the low-energy pions being reduced more rapidly. We approximate curve 2 by two exponential functions with different attenuation lengths for the depth intervals of 0 to $40 \mathrm{~g} / \mathrm{cm}^{2}$ and $>40 \mathrm{~g} / \mathrm{cm}^{2}$. The numerical values are listed in Table 1. For a 4.5-Gv cutoff, we obtain an attenuation profile for the low-energy pion production rate that closely resembles curve 1 . This indicates that the shape of the pion production spectrum at $4.5 \mathrm{Gv}$ in 1968 is independent of atmospheric depth.

Curve 3 in Figure 2 shows the depth dependence $D_{\text {co11 }}(E, x)$ for the production rate of knock-on negatrons, calculated in the one-dimensional approximation for particles traveling vertically downward. The one-dimensional approximation is applicable, since knock-ons in the energy range considered here are strongly collimated in the forward direction. The fast initial decay of curve 3 results from the rapid attenuation of multiply charged nuclei. Deeper in the atmosphere, production of knock-on negatrons by muons is accounted for and causes the attenuation length to increase. We use the same depth dependence for knock-ons of all energies, neglecting a slight variation in the shape of the production spectrum with depth that might be caused by the changing composition of the parent particles. The attenuation lengths for the exponential approximation (dashed lines) are listed in Table 1. 
We now consider electrons and photons from the decay of charged and neutral pions. The relationship between the depth dependence of the pion production rate and the corresponding electron source is determined by the kinematics of the $\pi \rightarrow \mu \rightarrow e$ decay. We assume for the pion source the exponential attenuation derived above for discrete depth intervals:

$$
D_{\pi}\left(E_{\pi}, x\right)=\exp \left[-x / \Lambda_{\pi}\left(E_{\pi}\right)\right]
$$

The attenuation of the electron production rate is derived with the following simplifying assumptions (which, however, do not seriously affect the results of the later calculations). The decay electrons are assumed to travel in the same direction as the parent pions; the lifetime of the pion is neglected compared to that of the muon, i.e., we assume $\tau_{\pi}=0$; and pions of total energy $E_{\pi}+m_{\pi} c^{\mathrm{a}}$ are assumed to result in monoenergetic electrons with energy

$$
E_{\star} \approx\left(E_{\pi}+m_{\pi} c^{2}\right) / 4
$$

The $\pi \rightarrow \mu \rightarrow e$ decay in the atmosphere has been discussed in detail by Verma [1967]. It is seen that the attenuation of the electron production rate with atmospheric depth $x$ is of the form

$$
\begin{aligned}
D_{\theta}\left(E_{e}, x\right) & \propto\left(\eta / x^{\eta+1}\right) \\
& \cdot \int_{0}^{x} \exp \left[-x_{1} / \Lambda_{\pi}\left(E_{\pi}\right)\right] x_{1}^{\eta} d x_{1}
\end{aligned}
$$

if the pion production rate decays as in (7). (The term $\eta x_{1}^{\eta} d x / x^{\eta+1}$ is the probability that a muon produced at depth $x_{1}$ will decay in $d x$ at $x$ $\left[\right.$ Verma, 1967]. Note that a pion lifetime $\tau_{\pi}=0$ has been assumed.) The parameter $\eta$ depends on the scale height of the atmosphere and on the lifetime and the momentum of the muon. One finds that

$$
\eta=1.08 / p_{\mu}=1.08\left(m_{\pi} / m_{\mu}\right) / p_{\pi}
$$

where $p_{\mu}$ and $p_{\pi}$ are the muon and pion momentum, and $m_{\mu}$ and $m_{\pi}$ are the corresponding rest masses. Evaluation of the integral in (9) shows that an approximate solution for the depth dependence of the electron production rate is given by

$$
D_{\bullet}\left(E_{\bullet}, x\right)=\exp \left[-\frac{\eta+1}{\eta+2} \frac{x}{\Lambda_{\pi}\left(E_{\pi}\right)}\right]
$$

This approximate solution does not deviate by more than $15 \%$ from the exact solution for the depth dependence at all energies. between $4 \mathrm{Mev}$ and $10 \mathrm{Gev}$ and for atmospheric depths between zero and $400 \mathrm{~g} / \mathrm{cm}^{2}$. The factor $r=(\eta+2) /$ $(\eta+1)$ varies between 1 and 2 for the nonrelativistic and the relativistic case, respectively. We note that for $r=2$ equation 10 reduces to (6). For photons we have, as a result of the immediate decay of the neutral pions,

$$
D_{\gamma}\left(E_{\gamma}, x\right)=\exp \left(-x / \Lambda_{\pi}\left(E_{\pi}\right)\right)
$$

with

$$
E_{\gamma} \approx\left(E_{\pi}+m_{\pi} c^{2}\right) / 2
$$

\begin{tabular}{|c|c|c|c|c|c|}
\hline \multirow[b]{3}{*}{ Particle Species } & \multirow{3}{*}{$\begin{array}{c}\text { Kinetic Energy, } \\
\text { Mev }\end{array}$} & \multicolumn{4}{|c|}{ Attenuation Length, $\mathrm{g} / \mathrm{cm}^{2}$} \\
\hline & & \multicolumn{2}{|c|}{$<0.5-\mathrm{Gr}$ Cutoff } & \multicolumn{2}{|c|}{ 4.5-Gv Cutoff } \\
\hline & & $x=0-40$ & $x>40$ & $x=0-40$ & $x>40$ \\
\hline \multirow[t]{2}{*}{ Pions } & $\leq 200$ & 67 & 105 & 120 & 120 \\
\hline & $\geq 10,000$ & 120 & 120 & 120 & 120 \\
\hline \multirow[t]{2}{*}{$\pi \rightarrow \mu \rightarrow e$ electrons } & $\leq 100$ & 76 & 120 & 137 & 137 \\
\hline & $\geq 4,000$ & 240 & 240 & 240 & 240 \\
\hline \multirow[t]{2}{*}{$\pi^{0} \rightarrow 2 \gamma$ photons } & $\leq 100$ & 67 & 105 & 120 & 120 \\
\hline & $\geq 4,000$ & 120 & 120 & 120 & 120 \\
\hline Knock-on negatrons & $\geq 4$ & 65 & 300 & 65 & 300 \\
\hline
\end{tabular}

Thus with the attenuation length $\Lambda_{\pi}$ for a given pion energy $E_{\pi}$, the electron and photon production rates have corresponding attenuation lengths

$$
\begin{aligned}
\Lambda_{e}\left(E_{\varepsilon}\right) & =\frac{\eta+2}{\eta+1} \Lambda_{\pi}\left(E_{\pi}\right) \\
\Lambda_{\gamma}\left(E_{\gamma}\right) & =\Lambda_{\pi}\left(E_{\pi}\right)
\end{aligned}
$$

TABLE 1. Attenuation Length $\Lambda$ for Production Rates 
where $E_{0}$ and $E_{\gamma}$ are given by (8) and (12). Above we have derived the depth dependence of the pion production rate for pions with energies above $\sim 10 \mathrm{Gev}$ and for pions below $\sim 200$ Mev. The decay of these pions yields electrons and photons with energies approximately above $4 \mathrm{Gev}$ and below $100 \mathrm{Mev}$. The corresponding attenuation lengths for the electron and the photon source are listed in Table 1 for the two intervals of 0 to $40 \mathrm{~g} / \mathrm{cm}^{2}$ and $>40 \mathrm{~g} / \mathrm{cm}^{2}$ atmospheric depth. For intermediate energies we perform an appropriate interpolation. It can be shown from (9) and considerations concerning the geometry of pion production that $\Lambda_{e}$ and $\Lambda_{\gamma}$ may be varied approximately as $\log E$ between $100 \mathrm{Mev}$ and $4 \mathrm{Gev}$. With the results of this section and the preceding section, we have thus determined the production rates of electrons and photons as functions of energy and atmospheric depth.

\section{Transport Equations}

In this section we present the one-dimensional transport equations for atmospheric secondary negatrons, positrons, and photons traveling vertically downward. The use of the one-dimensional approximation for the development of electromagnetic cascades in air has been justified by Butcher and Messel [1960]. The calculations refer to the flux of primary cosmic rays in summer 1968 as given for protons and heavier nuclei by (2) to (4). Although the major fraction of the secondary flux originates in interactions of cosmic-ray nuclei with air nuclei, secondary photons and electrons are also produced by primary and reentrant albedo electrons. In Figure 3 we show the differential kinetic energy spectra of electrons incident normally on the top of the atmosphere. Data points are for reentrant albedo electrons as measured in 1967 by Israel [1969] at Palestine, Texas. The point at high energies is by Daniel and Stephens [1967] for Hyderabad. The solid curve represents our best estimate of the spectrum of reentrant albedo electrons near Palestine in 1968. The dashed curve in Figure 3 represents the idealized spectrum of galactic electrons as derived from data available in early 1968 [Cline et al., 1964; L'Heureux, 1967; Daniel and Stephens, 1967; Webber and Chotkowski, 1967; Bleeker et al., 1968; Fanselow, 1968; L'Heureux and 'Meyer, 1968;
Webber, 1968; Israel, 1969]. This spectrum is used in the present calculations for the case of zero cutoff, although more recent measurements [Simnett and McDonald, 1968; Beuermann et $a l ., 1969 b]$ indicate a different spectral shape below $30 \mathrm{Mev}$ and a reduced intensity in the 30- to 200-Mev interval (shaded area). Note, however, that only a negligible effect on the total flux of atmospheric secondary electrons arises from these differences.

Consider now the transport of atmospheric secondary negatrons, positrons, and photons, as well as of residual primary electrons with differential (kinetic) energy spectra $f^{-}(E, x), f^{+}(E, x), g(K, x)$, and $p(E, x)$, respectively. $E$ and $K$ are the charged-particle kinetic energy and the photon energy, respectively, and $x$ is the vertical atmospheric depth. Within the one-dimensional approximation of electromagnetic cascade theory [Rossi, 1952], the transport in energy and space is described by a set of diffusion equations:

$$
\begin{gathered}
\frac{\partial f^{-}(E, x)}{\partial x}=-\frac{\partial}{\partial E}\left[\frac{d E}{d x} f^{-}(E, x)\right] \\
+\int_{E}^{\infty} g(K, x) \sigma_{p}(K, E) d K \\
+a g(a E, x) \sigma_{c}(a E) \\
+Q_{\bullet}-(E, x)+Q_{\mathrm{col1}}(E, x) \\
+\int_{2 E}^{\infty}\left[f^{-}\left(E^{\prime}, x\right)+f^{+}\left(E^{\prime}, x\right)\right. \\
\left.+p\left(E^{\prime}, x\right)\right] \sigma_{\mathrm{col1}}\left(E^{\prime}, E\right) d E^{\prime} \\
\frac{\partial f^{+}(E, x)}{\partial x}=-\frac{\partial}{\partial E}\left[\frac{d E}{d x} f^{+}(E, x)\right] \\
+\int_{E}^{\infty} g(K, x) \sigma_{p}(K, E) d K \\
+Q_{\bullet}(E, x) \\
\frac{\partial g(K, x)}{\partial x}=-\mu(K) g(K, x) \\
+\int_{K}^{\infty}\left[f(E, x)+f^{+}(E, x)\right. \\
+p(E, x)] \sigma_{r}(E, K) d E \\
+\frac{a}{a-1} g\left(\frac{a K}{a-1}, x\right) \sigma_{c}\left(\frac{a K}{a-1}\right) \\
+Q_{\gamma}(K, x)
\end{gathered}
$$




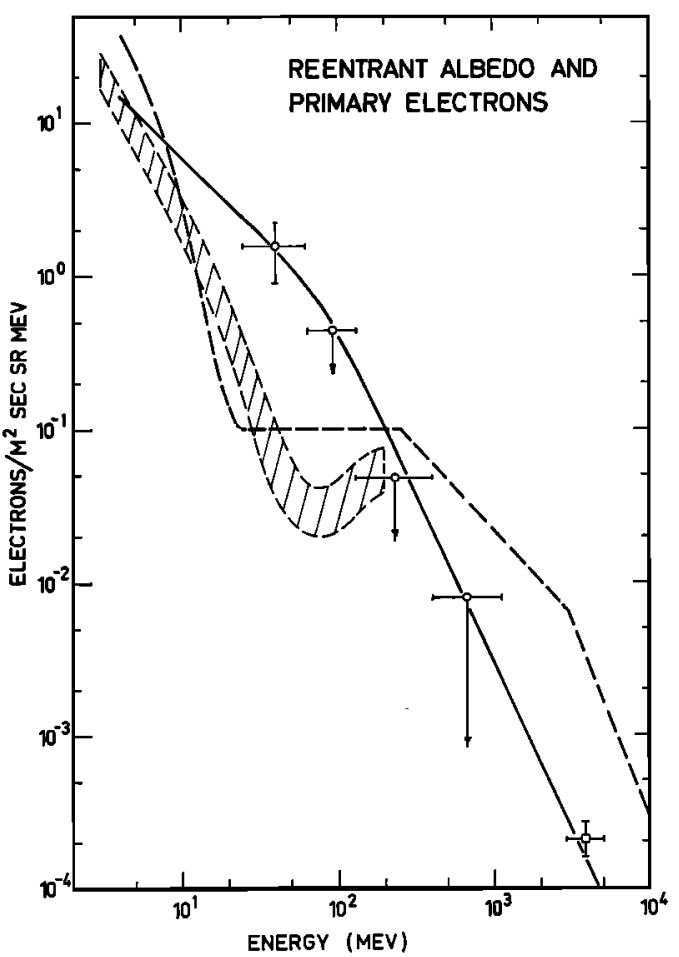

Fig. 3. Differential energy spectra of primary electrons (dashed curve) and reentrant albedo electrons (solid curve) used for the present calculations. The data points are for reentrant albedo electrons observed in 1967 near Palestine, Texas, by Israel [1969] (open circles) and near Eyderabad, India, by Daniel and Stephens [1967] (open square). The shaded area indicates the shape of the primary electron spectrum according to recent measurements by Simnett and McDonald [1968] and Beuermann et al. [1969a, b].

$$
\frac{\partial p(E, x)}{\partial x}=-\frac{\partial}{\partial E}\left[\frac{d E}{d x} p(E, x)\right]
$$

The first term in each of the four equations is the loss term, e.g., $\mu(K)$ in (15) is the total attenuation coefficient for photons of energy $K$. For charged particles, the loss term $d E / d x$ includes both ionization and bremsstrahlung. The latter is treated as continuous energy degradation rather than as a discontinuous process. This simplifies the calculations considerably and does not seriously affect the results at low energies. The first integral terms in (13) and (14) represent pair production with a differential cross section $\sigma_{p}(K, E)$ for a photon of energy $K$ to produce an electron of energy $E$. In (15), correspondingly, we have bremsstrahlung as photon source with the differential cross section $\sigma_{r}(E, K)$. The second integral in (13) represents the contribution to the knock-on source related to electron-electron collisions, with the differential cross section $\sigma_{\text {coll }}\left(E^{\prime}, E\right)$ for a positron or negatron of energy $E^{\prime}$ to produce a knock-on negatron of energy $E$. The differential cross sections as given by Rossi [1952] are used. Only slight approximations have been performed in some cases to simplify the calculations. The Compton effect, being of minor importance, is treated in an approximate way. The assumption is made that photons of energy $K$ result in monoenergetic negatrons of kinetic energy $E=K / a$, with $a=1.26$. (Though the mean energy of all Compton negatrons in units of the photon energy varies with $K$, the above assumption is met rather well down to $K=5 \mathrm{Mev}$ with a cross section $\sigma_{c}(K)$ redefined as the probability for a photon of energy $K$ to produce a negatron of $E>3 \mathrm{Mev})$. The terms $Q_{\mathrm{e}}+(E, x), Q_{\mathrm{o}}-(E, x)$, and $Q_{\gamma}(K, x)$ are the production spectra of positrons, negatrons, and photons from decaying pions, as was discussed in the two preceding sections. To obtain $Q_{0}+$ and $Q_{0}-$ from the production spectrum of decay electrons shown in Figure 1 , we adopt a charge ratio of positrons to negatrons of 1.25 , independent of energy and atmospheric depth. This value has been determined as the best estimate from observations of the muon spectra in the atmosphere [MacKeown and Wolfendale, 1966]. $Q_{\text {coll }}(E, x)$ is the production spectrum of knock-on negatrons related to the nucleonic component, as given in Figure 1 and Table 1.

At $x=0$, initial conditions are specified for the production spectra $Q_{v}(E, 0)$ and the spectrum of electrons incident on the top of the atmosphere, $p(E, 0)$; the initial intensities of secondary electrons and of primary and secondary photons are set equal to zero. We then determine separately the two contributions to the total secondary flux related (1) to cosmic ray nuclei via the $Q_{i}(E, x)$ and (2) to reentrant albedo and galactic electrons via $p(E, 0)$.

The transport equations 13 to 16 are integrated for energies $E, K>4 \mathrm{Mev}$. For the range of $4 \mathrm{Mev}$ to $4 \mathrm{Gev}$, they are solved numerically by stepwise integration. At energies above $4 \mathrm{Gev}$, we use the analytical solutions of cascade theory [Rossi, 1952]. Let us 
first consider the numerical part for energies below 4 Gev. Starting at the top of the atmossphere, the spectra are simultaneously propagated downward in steps of $0.1 \mathrm{~g} / \mathrm{cm}^{2}$ to a depth of $40 \mathrm{~g} / \mathrm{cm}^{2}$. At this depth the values of the attenuation lengths for the production rates are changed (see Table 1). The integration of (13) to (16) is then continued with a step width of $0.5 \mathrm{~g} / \mathrm{cm}^{2}$ to a depth of $400 \mathrm{~g} / \mathrm{cm}^{2}$, i.e., well through the transition maximum. Tests carried out with different step widths indicate that cumulative errors stay below $3 \%$ at any depth and energy.

Above $4 \mathrm{Gev}$, we use the stationary solutions in approximation $A$ of electromagnetic cascade theory. These solutions have frequently been discussed in the literature [e.g., Rossi, 1952;
Okuda and Yamamoto, 1965]. Here we only note that energy loss by ionization, the Compton effect, and the production of knock-on negatrons are neglected in approximation $A$, whereas bremsstrahlung is correctly treated as a discontinuous process; the stationary solutions apply when the production spectra are power laws in energy. Above $4 \mathrm{Gev}$, we approximate the production spectra shown in Figure 1 by powerlaw spectra with exponents -2.6 and -3.6 for photons and electrons, respectively. We then obtain analytical solutions for the spectra $f^{-}(E$, $x), f^{+}(E, x), g(K, x)$, and $p(E, x)$, valid above $4 \mathrm{Gev}$ and at all depths. They provide a quite accurate description of the cascade development in the high-energy region. At $4 \mathrm{Gev}$, the numerical solution does not necessarily fit the
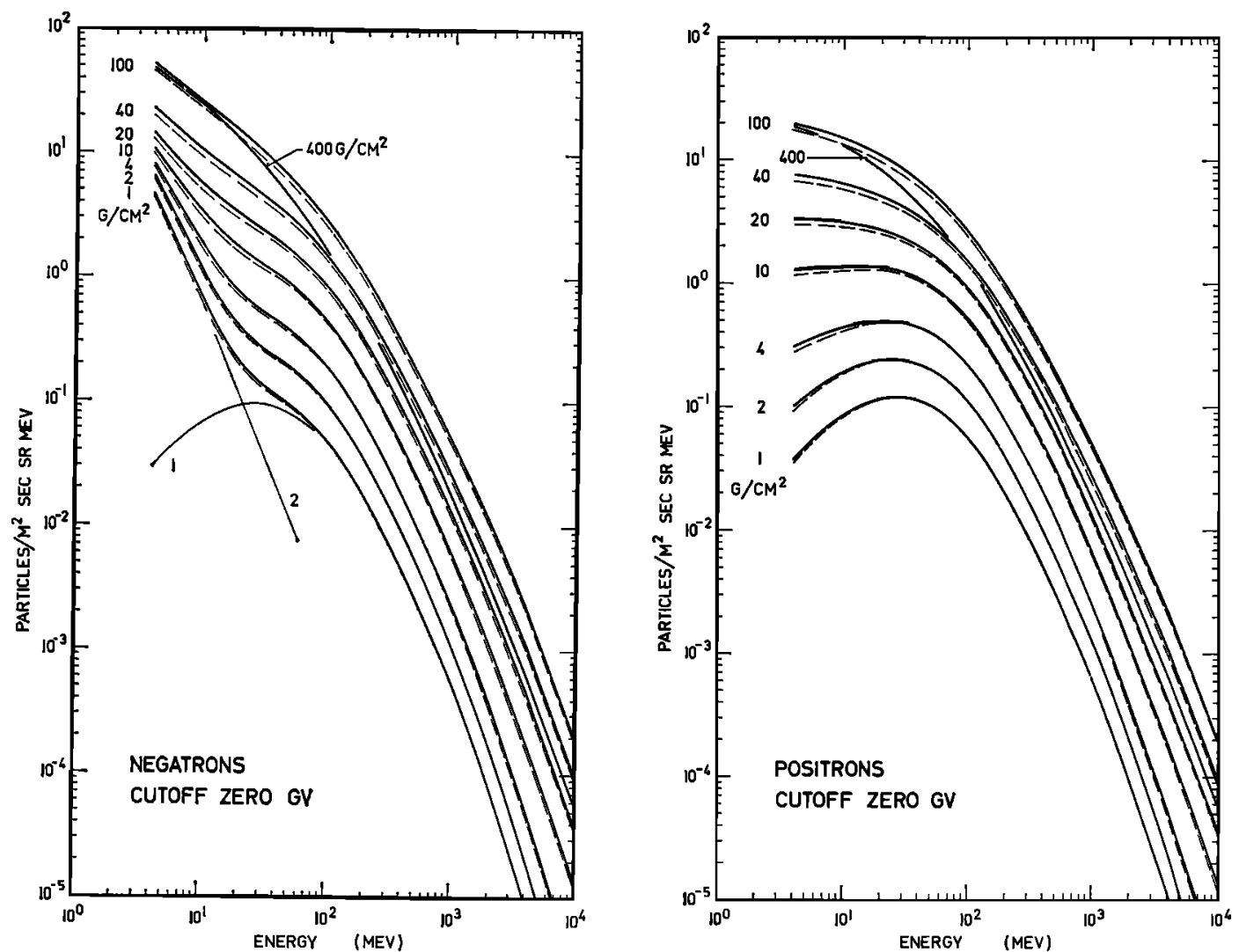

Fig. 4. Calculated differential kinetic energy spectra of downward-moving secondary negatrons and positrons at atmospheric depths between 1 and $400 \mathrm{~g} / \mathrm{cm}^{2}$ for 1968 and for zero geomagnetic cutoff. The dashed curves represent negatrons and positrons produced by the cosmic ray nucleonic component; the solid curves, in addition, include secondary negatrons and positrons that originate from cascades initiated by primary electrons. Curves 1 and 2 indicate the contributions to the negatron flux at a depth of $1 \mathrm{~g} / \mathrm{cm}^{2}$ from the $\pi^{-} \rightarrow \mu^{-} \rightarrow e^{-}$decay and the knock-on source. 
analytical one. A discrepancy may develop with increasing atmospheric depth, since the energy losses of electrons by bremsstrahlung and ionization are treated differently at energies above and below $4 \mathrm{Gev}$. After each integration step we therefore adjust the numerical solution at $4 \mathrm{Gev}$ to the value obtained from the stationary solution at this energy. This provision minimizes errors that might accrue from our low-energy approximation of the bremsstrahlung process.

\section{Results and Discussion}

In this section, we present numerical results on the energy spectra and the depth dependence of atmospheric secondary negatrons, positrons, and photons in 1968 for energies between 4 $\mathrm{Mev}$ and $10 \mathrm{Gev}$ and atmospheric depths ranging from 1 to $400 \mathrm{~g} / \mathrm{cm}^{2}$. The results on the attenuation of residual primary electrons, $p(E, x)$, are not given here, since they depend critically on the assumed primary spectrum.

Electron spectra. Figure 4 shows the differential kinetic energy spectra of negatrons $f^{-}(E, x)$ and of positrons $f^{+}(E, x)$ in the vertical direction for a zero geomagnetic cutoff. The major fraction of the secondary flux originates from collisions of primary cosmic ray nuclei. This flux is represented by the dashed curves. The solid curves include the secondaries that arise from the primary cosmic ray electron flux, $p(E, 0)$ (Figure 3) impinging on the top of the atmosphere. In general, the contribution from primary electrons to the total secondary flux is small, $15 \%$ or less. The ratio of electron-produced to nucleon-produced secondaries varies with depth, as is indicated in Figure 6 below.

In Figure 4, the contributions from the knockon source, $Q_{\text {conl }}(E, x)$, and the pion source, $Q_{e}-(E, x)$, to the negatron flux at $1 \mathrm{~g} / \mathrm{cm}^{2}$ are separately indicated by thin lines. The marked difference in the spectral shapes of negatrons and positrons in the high atmosphere is due to the abundant production of knock-on negatrons by cosmic ray nuclei. The spectral shape at $1 \mathrm{~g} / \mathrm{cm}^{2}$ (after ten integration steps) still resembles that of the production spectra. With increasing depth, the energy loss by ionization strongly influences the propagation of the lowenergy particles and causes a rapid change of the spectral shape. At depths exceeding one radiation length, $37 \mathrm{~g} / \mathrm{cm}^{2}$, the shape stabilizes. It is now increasingly determined by pair produc-

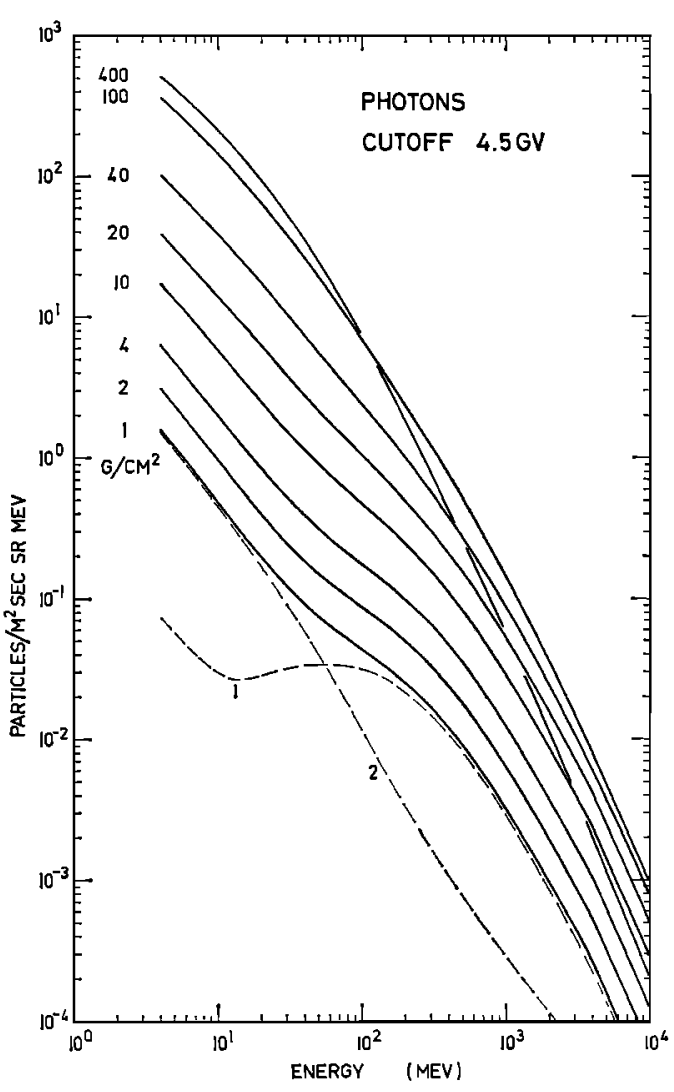

Fig. 5. Calculated differential energy spectra of downward-moving atmospheric secondary photons at depths between 1 and $400 \mathrm{~g} / \mathrm{cm}^{2}$, for 1968 and 4.5-Gv geomagnetic cutoff. The solid curves give the flux of secondary photons from interactions of cosmic ray nuclei and cascades initiated by primary and reentrant albedo electrons. The dashed curves indicate the contributions to the photon flux at $1 \mathrm{~g} / \mathrm{cm}^{2}$ from cosmic ray nuclei (curve 1) and from primary and reentrant albedo electrons (curve 2).

tion, the Compton effect, and electron-electron collisions. The latter two processes cause a persistent excess of negatrons over positrons at energies below $100 \mathrm{Mev}$. At great depth, $>200 \mathrm{~g} / \mathrm{cm}^{2}$, the spectral shape finally assumes an equilibrium state. Here the cascade processes dominate, and direct production of electrons from $\pi \rightarrow \mu \rightarrow e$ decays is of minor importance. The equilibrium spectrum is therefore expected to resemble the energy distribution in electromagnetic cascades. Indeed, we find that the electron (i.e., negatron plus positron) spectrum at $400 \mathrm{~g} / \mathrm{cm}^{2}$ is in good agreement with the track length distribution calculated by Richards and 
Nordheim [1948], i.e., with the average cascade spectrum.

Photon spectra. Figure 5 shows the differential energy spectra of photons in the vertical direction for a geomagnetic cutoff of $4.5 \mathrm{Gv}$, corresponding to Palestine, Texas. At this latitude many observations of the atmospheric secondary photon flux have been made in connection with a search for galactic photons. The most striking feature of the atmospheric photon spectrum is the absence of a pronounced peak at $68 \mathrm{Mev}$ from the decays of neutral pions. The spectrum rises continually to low energies as a result of the abundant bremsstrahlung production by reentrant albedo electrons. For a depth of $1 \mathrm{~g} / \mathrm{cm}^{2}$, the two contributions from primary cosmic ray nuclei (curve 1) and reentrant albedo and primary electrons (curve 2) are plotted separately as dashed lines. Near the expected position of the $\pi^{0}$ peak, both sources are approximately of equal strength. The rising part of curve 1 below $\sim 10 \mathrm{Mev}$ represents the initial stage of the cascade development, namely bremsstrahlung photons from $\pi \rightarrow \mu \rightarrow e$ decay electrons, superimposed on the $\pi^{0} \rightarrow 2 \gamma$ spectrum. The flux of these second-generation photons increases approximately as the square of the depth and dominates the bremsstrahlung photons from reentrant albedo electrons already at $\sim 10 \mathrm{~g} / \mathrm{cm}^{2}$ (compare Figure 6 ).
Depth dependence. Consider now the functional form of the intensity versus depth curves (growth curves). The shape of these curves is energy dependent, reflecting the variation of the spectral shape with depth. In Figure 6 we present sample growth curves for the flux of secondary electrons and photons at four selected energies between $10 \mathrm{Mev}$ and $10 \mathrm{Gev}$, calculated for a zero geomagnetic cutoff. Electrons are represented by solid curves, photons by dashed curves. We plot the fluxes of secondaries related to cosmic ray nuclei and to primary electrons as thick and thin curves, respectively. Distinctly different shapes are obtained for the growth curves of these two components of the secondary flux. Both influence the depth dependence of the total secondary flux by their relative magnitudes. As was discussed above, electron-produced secondaries generally represent a rather small contribution only. The depth dependence of the total flux will then be similar to that of the secondaries from collisions of cosmic-ray nuclei. For low-energy photons, however, electron-produced secondaries, i.e., bremsstrahlung photons, dominate in the high atmosphere. The composite growth curve for $10-\mathrm{Mev}$ photons is shown as a dotted curve.

For both components, the functional form of the growth curve for a given energy generally depends on the characteristics of more than one

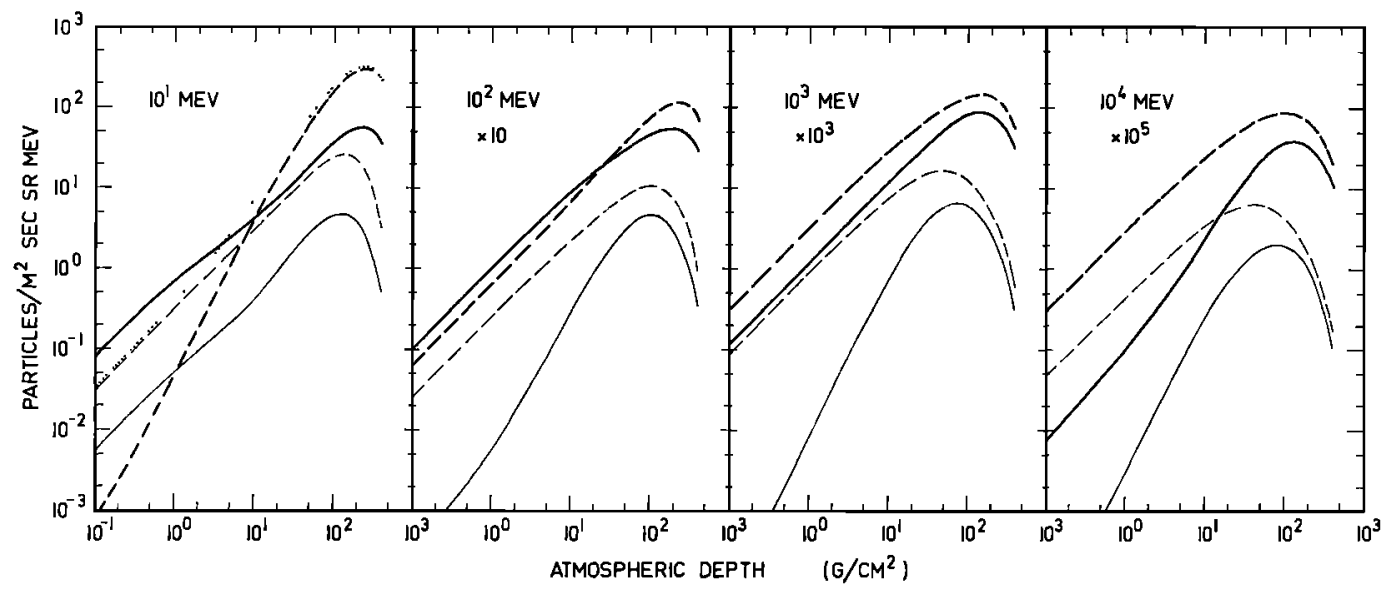

Fig. 6. Growth curves for the differential flux of secondary electrons and photons with energies between $10 \mathrm{Mev}$ and $10 \mathrm{Gev}$, calculated for 1968 and zero geomagnetic cutoff. The thick curves indicate the flux of electrons (solid) and photons (dashed) from interactions of cosmic ray nuclei; the corresponding thin curves give the flux of secondaries produced in cascades of primary electrons (not including the residual primary electron itself). For photons of $10 \mathrm{Mev}$, the total flux has been indicated by a dotted curve. 
particle source. The bulging shape of the two curves shown for 10-Mev electrons, for example, is caused by the knock-on negatrons approaching equilibrium near $10 \mathrm{~g} / \mathrm{cm}^{2}$ atmospheric depth and the low-energy cascade electrons picking up near $30 \mathrm{~g} / \mathrm{cm}^{2}$. The superposition of these components leads to a complicated functional depth dependence of the total secondary flux.

Consider now the problem of separating a small primary cosmic ray flux from an abundant atmospheric background. This problem is encountered in the determination of the extraterrestrial electron flux below $\sim 500 \mathrm{Mev}$ at balloon altitude. A technique used by various investigators is to express the measured flux as a function of atmospheric depth by a linear combination of two curves, one describing the growth of the secondary component and the other the attenuation of the residual primary flux. It is commonly assumed that, at low energies, the atmospheric flux grows linearly with depth [Beedle and Webber, 1968; Israel and Vogt, 1968]. For energies $>300 \mathrm{Mev}$, L'Heureux and Meyer [1968] use the functional depth dependence $A x+B x^{2}$, where the quadratic term allows for a contribution of pair-produced electrons of the second generation. We find that our calculated growth curves display this behavior in the very high atmosphere only. Deviations from a linear or quadratic growth of the relevant components occur already at atmospheric depths of a few $\mathrm{g} / \mathrm{cm}^{2}$ or less. For example, the linear growth of $10-\mathrm{Mev}$ electrons is maintained only at depths $<0.5 \mathrm{~g} / \mathrm{cm}^{2}$. Thus a more accurate fitting procedure must take the deviations from a linear growth into account.

We have approximated the functional depth dependence of the total secondary flux (related to cosmic ray nuclei and to primary electrons) by power laws in $x$ for two discrete depth intervals:

$$
\begin{aligned}
& f_{1}(E, x) \propto x^{n_{1}(E)} \text { for } 2 \leq x \leq 15 \mathrm{~g} / \mathrm{cm}^{2} \\
& f_{2}(E, x) \propto x^{n_{2}(E)} \text { for } 15 \leq x \leq 40 \mathrm{~g} / \mathrm{cm}^{2}
\end{aligned}
$$

where $E$ is the energy of the secondary particles, and $f_{1}(E, 15)=f_{2}(E, 15)$. In Figure 7 , we plot the power-law exponents $n_{1}$ and $n_{2}$ versus energy as solid and dashed curves. The curves for electrons and, separately, for negatrons and positrons are for 1968 and a zero geomagnetic cutoff. The photon curves are for 1968 and a
4.5-Gv cutoff. The deviations from a linear growth $(n=1)$ are clearly evident. They are most pronounced at the lowest energies. Detailed features of the curves are real, established by ten values of $n$ calculated per decade in energy. Using this power-law approximation of the functional depth dependence will facilitate fitting the expected atmospheric secondary flux to a measured growth curve. Since $n_{1}<1$ for electrons with energies below $500 \mathrm{Mev}$, the derived primary flux will be slightly lower than with the assumption of a linear growth of the secondary flux.

Finally, we are interested in the general applicability of the derived functional depth dependence. We investigate the solar cycle variation of the $\log -\log$ slopes of the growth curves, $n_{1}$ and $n_{2}$, by repeating our calculations for the time of solar minimum and for a zero geomagnetic cutoff. We use the 1965 production spectra of secondary electrons and photons of Perola and Scarsi [1966] (personal communication, G. C. Perola), the 1965 cosmic ray spectra of Ormes and Webber [1966], and the primary electron spectrum shown in Figure 3. Although the flux of low-energy secondaries changes by a factor of 2 between 1965 and 1968, there is little difference between the slopes of the growth curves for the two years. For secondary electrons of selected energies, we list, in Table 2, the deviations $\Delta n_{1}$ and $\Delta n_{2}$ of the 1965 slopes from those for 1968, as given in Figure 7. In the next two sections, comparisons of our calculated 1968 energy spectra with experimental data reveal inaccuracies in the assumed production spectra of secondaries from the decays of charged and neutral pions, as shown in Figure 1 . However, it is found that an adjustment of the production spectra does not affect the functional depth dependence of the secondary flux to any significant extent.

Though the absolute secondary flux in the high atmosphere is proportional to the production rate, we find that the functional depth dependence of the secondary flux is insensitive to moderate variations of the production rates. Only this functional dependence of secondaries upon depth, and not their absolute flux, is used in separating primary and secondary fluxes. Our results on the depth dependence are therefore also usable with data for years other than 1968. 

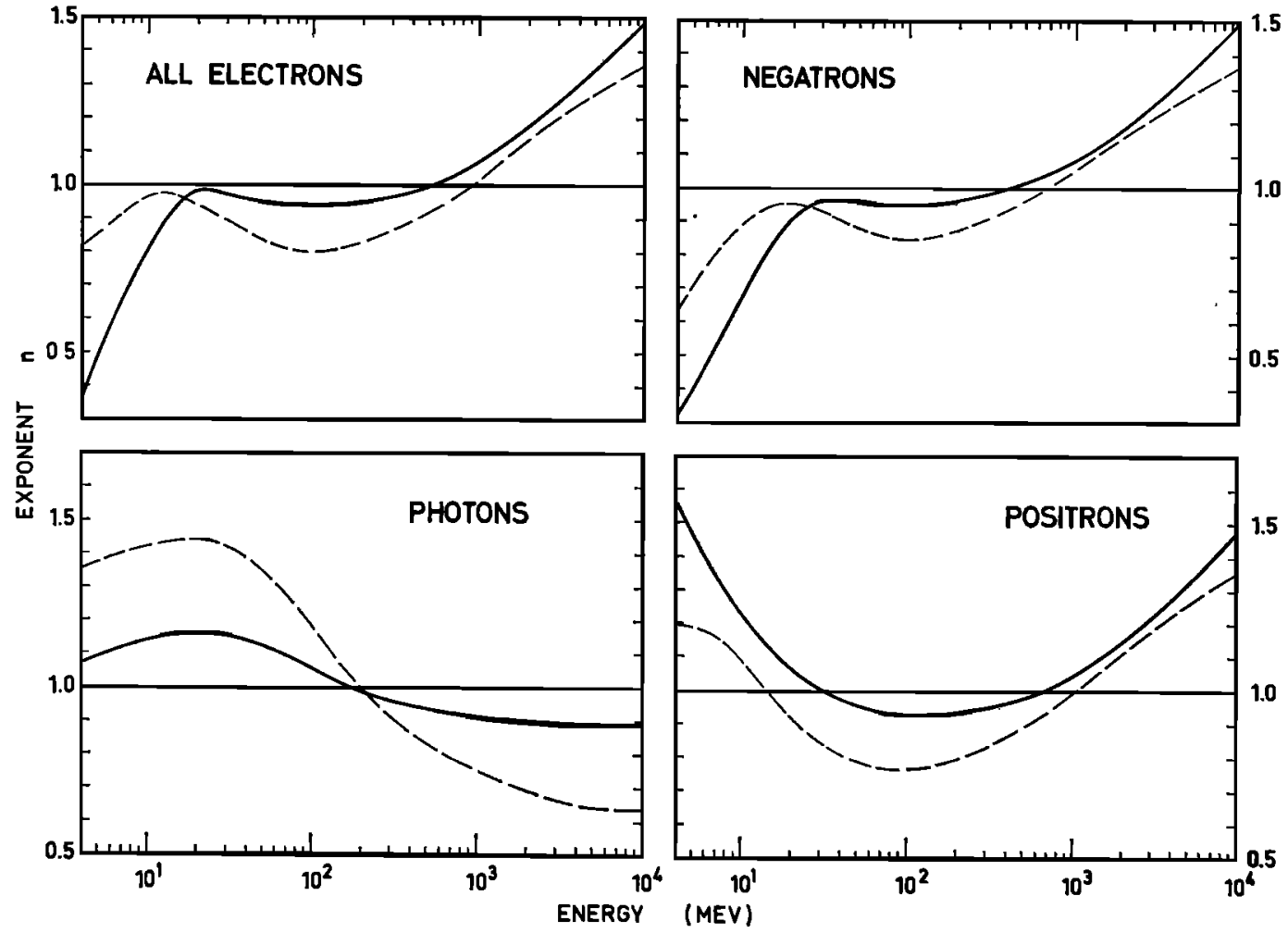

Fig. 7. The exponent $n_{i}$ of a power law fit $f(x) \propto x^{n i}$ to the growth curves of the differential atmospheric secondary flux as a function of energy. Solid curves give $n_{1}$ for a fit to the 2- to 15-g/ $\mathrm{cm}^{2}$ depth interval, dashed curves give $n_{2}$ for the $15-$ to $40-\mathrm{g} / \mathrm{cm}^{2}$ interval. The curves for electrons are calculated for zero geomagnetic cutoff; the curves for photons are for 4.5-Gv cutoff.

\section{Comparison with 1968 Electron Data}

In this section, we compare the calculated growth curves and energy spectra with our own data on atmospheric secondary negatrons and positrons. Utilizing a balloon-borne magnetic spectrometer, we measured negatrons and pos-

TABLE 2. Deviations of $n_{1}$ and $n_{2}$ for Secondary Electrons from Values Given in Figure 7

\begin{tabular}{rrrrrr}
\hline & \multicolumn{2}{c}{$\begin{array}{c}1965 \text { (Perola } \\
\text { and Scarsi) }\end{array}$} & & \multicolumn{2}{c}{1968 Adjusted } \\
\cline { 2 - 3 } \cline { 5 - 6 } Energy, Mev & \multicolumn{1}{c}{$\Delta n_{1}$} & \multicolumn{1}{c}{$\Delta n_{2}$} & & \multicolumn{1}{c}{$\Delta n_{1}$} & \multicolumn{1}{c}{$\Delta n_{2}$} \\
\hline 4 & 0.09 & -0.04 & & -0.02 & -0.16 \\
10 & 0.07 & -0.02 & & -0.06 & -0.06 \\
40 & -0.02 & -0.06 & & -0.01 & 0.00 \\
100 & -0.02 & -0.07 & & 0.00 & 0.01 \\
400 & -0.02 & -0.05 & & 0.02 & 0.02 \\
10,000 & 0.00 & 0.00 & & 0.05 & -0.04 \\
\hline
\end{tabular}

itrons with momenta from 6 to $>200 \mathrm{Mv}$ in summer 1968 near Ft. Churchill, Canada. Information on the flights and a short description of the instrument have been given elsewhere [Beuermann et al., 1969a,b]. The ascent curves discussed below are derived from nighttime observations when no reentrant albedo electrons were present.

The data collected in the high atmosphere represent a mixture of primary cosmic ray electrons and atmospheric secondaries. The method used to separate the primary and the atmospheric secondary flux has been described by Israel and Vogt [1968] and Beuermann et al. $[1969 a, b]$. In short, the measured depth dependence of the positron or negatron flux $J_{i}{ }^{ \pm}\left(x_{j}\right)$, for an energy interval $i$ and atmospheric depths $x_{1}$, is least squares fitted by a linear combination of two curves:

$$
J_{i}{ }^{ \pm}\left(x_{i}\right) \simeq a_{i}{ }^{ \pm} f_{i}{ }^{ \pm}(x)+b_{i}{ }^{ \pm}{p_{i}}^{ \pm}(x)
$$


where $f_{t}^{ \pm}(x)$ is the calculated depth dependence of the atmospheric flux, and $p_{\imath}{ }^{ \pm}(x)$ is the depth dependence of the residual primary flux. In the present case, the parameters $a_{i}{ }^{ \pm}$and $b_{i}{ }^{ \pm}$are determined from a least squares fit of (17) to the measured fluxes at five depths $x$, between 2.4 and $42 \mathrm{~g} / \mathrm{cm}^{2}$. The atmospheric flux corrected for the primary contribution is given by

$$
F_{\imath}{ }^{ \pm}\left(x_{i}\right)=J_{\imath}{ }^{ \pm}\left(x_{1}\right)-b_{i}{ }^{ \pm} p_{i}{ }^{ \pm}\left(x_{1}\right)
$$

In comparing experimental and theoretical fluxes, we have to remember that $b_{i}{ }^{ \pm}$depends on $f_{i}^{ \pm}(x)$ via (17). For the data discussed below, however, the second term on the right side of (18) represents only $\sim 30 \%$ of the observed flux $J^{ \pm}(2.4)$ at the float altitude of $2.4 \mathrm{~g} / \mathrm{cm}^{2}$, and considerably less deeper in the atmosphere.

In Figure 8, we show the growth curves for atmospheric secondary negatrons and positrons of three selected energy intervals. The data points represent the observed fluxes $J_{i}{ }^{ \pm}\left(x_{j}\right)$ for $x_{j}>42 \mathrm{~g} / \mathrm{cm}^{2}$, and the corrected fluxes $F_{i}{ }^{*}\left(x_{j}\right)$ for $x_{3} \leq 42 \mathrm{~g} / \mathrm{cm}^{2}$. To indicate the magnitude of the correction for residual primary negatrons and positrons, we include, as phantom points, the observed fluxes $J^{ \pm}(2.4)$ at float altitude. The experimental data are compared with the curves of absolute intensity versus depth for secondaries from collisions of cosmic ray nuclei and from primary electrons, as calculated in the preceding sections for 1968 and a zero geomagnetic cutoff (solid curves). These curves are based upon the production spectra of Perola and Scarsi [1966], as shown in Figure 1. We use these curves of absolute intensity versus depth to represent $f_{i} \pm(x)$ in (17). The best-fit depth dependence $a_{i}{ }^{ \pm} f_{i}{ }^{ \pm}(x)$, as obtained from a fit of (17) to the observed fluxes, is represented by the dashed curves. Factors $a_{i}^{ \pm}$different from 1 indicate errors in the assumed production rates. For negatrons and positrons of 25 to 200 Mev, arising predominantly from $\pi \rightarrow \mu \rightarrow e$ decays, we obtain an average value $a^{ \pm}=0.75$. Thus the corresponding production rate of Perola and Scarsi (Figure 1) is in error by $\sim 25 \%$. We note that an error of this magnitude is consistent with the precision quoted by these authors for their theoretically derived secondary spectra. For knock-on negatrons of 6.5 to 12.5 $\mathrm{Mev}$, on the other hand, near agreement is
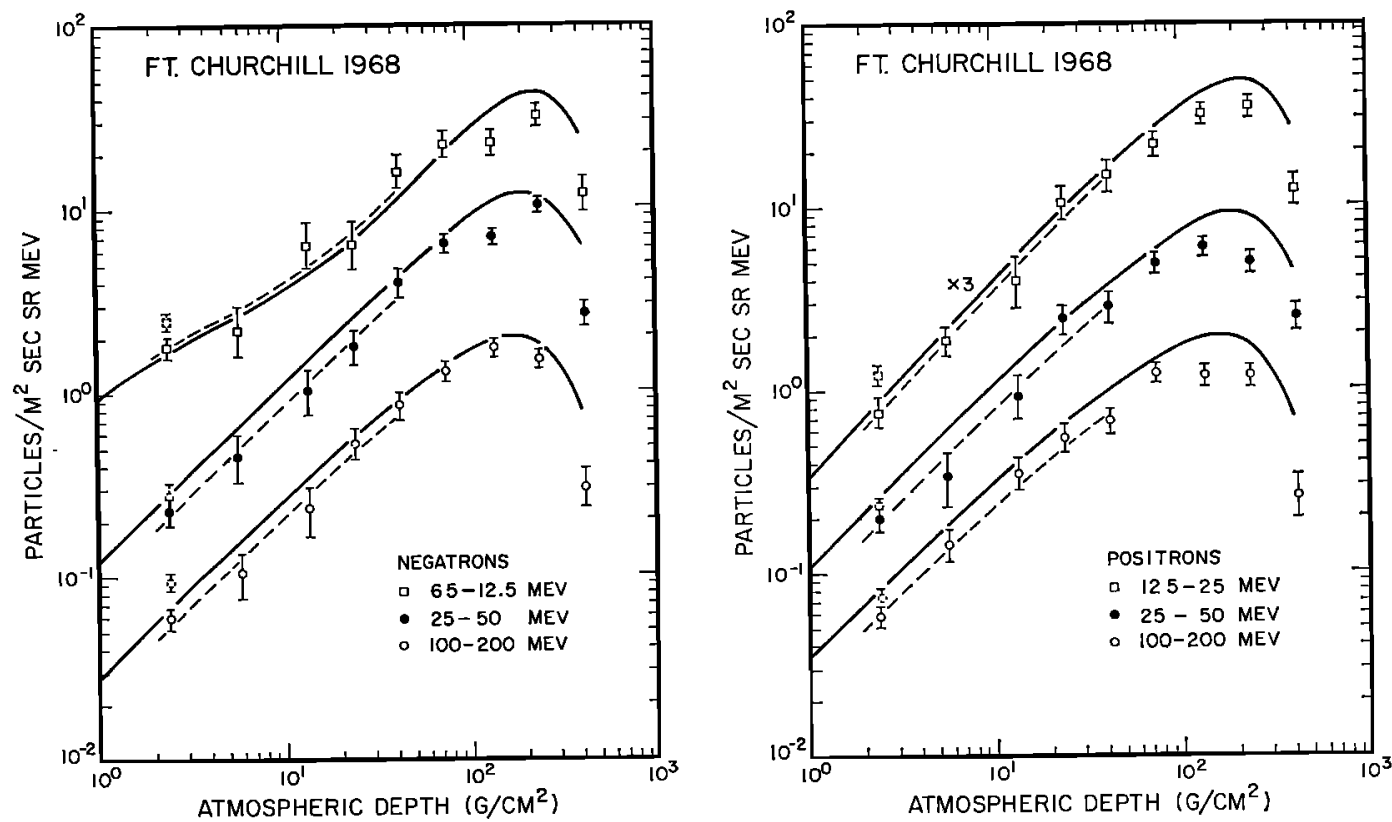

Fig. 8. The growth of atmospheric secondary negatrons and positrons in three selected energy intervals as derived from our measurements. The total flux measured at a depth of 2.4 $\mathrm{g} / \mathrm{cm}^{2}$ is indicated by phantom points. Also shown are the calculated depth dependence $f_{i}{ }^{*}(x)$ for the same energy intervals (solid curves) and the best fit depth dependence $a_{i}^{ \pm} f_{i}^{ \pm}(x)$ (dashed curves). 


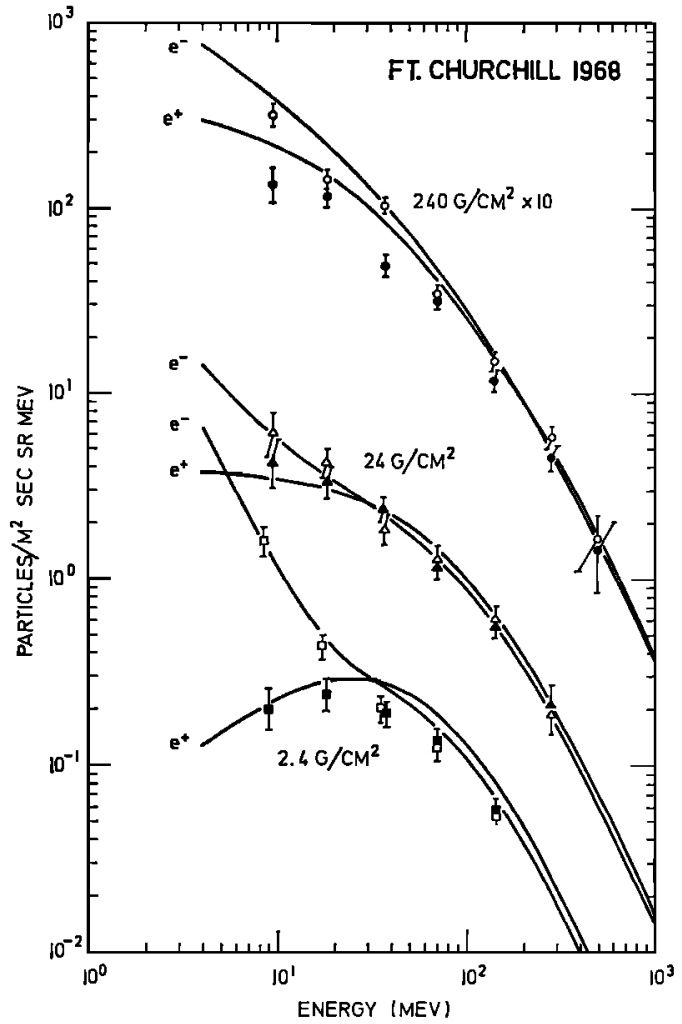

Fig. 9. Differential kinetic energy spectra of atmospheric secondary negatrons and positrons at 2.4-, 24-, and $240-\mathrm{g} / \mathrm{cm}^{2}$ depth as derived from our measurements in summer 1968 near Fort Churchill. Negatrons are indicated by open, positrons by closed symbols. Also shown are the calculated energy spectra for the same depths.

achieved between measured and calculated fluxes, confirming the production rate for this component (Figure 1).

If adjusted by the factor $a_{4}^{ \pm}$, the calculated curves excellently reproduce the measured intensity as a function of atmospheric depth. The agreement then extends beyond the data points used in the least squares fit to atmospheric depths near and past the transition maximum. This indicates that our general approach is appropriate to describe the propagation of lowenergy secondary electrons through the atmosphere. The agreement at great depths adds to our confidence in the derived functional depth dependence for $x \leq 40 \mathrm{~g} / \mathrm{cm}^{2}$, as depicted in Figure 7. The measurements in Figure 8 confirm the predicted difference in the depth dependence of low-energy negatrons and positrons.
The log-log slope of the experimental growth curve for 6.5 - to $12.5-\mathrm{Mev}$ negatrons is less than 1 , in agreement with the value given in Figure 7. On the other hand, the buildup of the 12.5 - to $25-\mathrm{Mev}$ positron flux proceeds, in fact, at a rate slightly exceeding a linear growth. An accurate determination of a small primary flux thus requires a careful consideration of the relevant growth rates.

In Figure 9, we present the differential kinetic energy spectra of negatrons and positrons at 2.4-, 24-, and $240-\mathrm{g} / \mathrm{cm}^{2}$ atmospheric depth. Data points are the same as in Figure 8; the solid curves represent the calculated energy spectra based upon the production spectra of Figure 1. The measurements confirm the predicted variation of the spectral shape with increasing atmospheric depth. In particular, the calculated spectra correctly describe the rapid attenuation of the intense flux of knock-on negatrons $<20 \mathrm{Mev}$ between 2.4 and $24 \mathrm{~g} / \mathrm{cm}^{2}$ atmospheric depth. The spectra measured under a residual atmosphere of $2.4 \mathrm{~g} / \mathrm{cm}^{2}$ permit a determination of the charge ratio of $\pi^{ \pm} \rightarrow \mu^{ \pm} \rightarrow$ $e^{ \pm}$decay electrons at production. After applying an appropriate correction for knock-on negatrons, we obtain an experimental value $N\left(e^{+}\right)$/ $N\left(e^{-}\right)=1.20 \pm 0.16$, in good agreement with the value of 1.25 assumed above.

\section{Photon Spectrum and High-Energy Production Rates}

A striking feature of the calculated photon spectrum (Figure 5) is the apparent non-existence of the $\pi^{0} \rightarrow 2 \gamma$ peak at $68 \mathrm{Mev}$. In an earlier section, we concluded that this peak is masked by the abundant flux of low-energy bremsstrahlung photons. This conclusion is substantiated by a comparison with the experimentally determined photon spectrum in the high atmosphere.

Flux and energy spectra of photons at atmospheric depths between 3 and $11 \mathrm{~g} / \mathrm{cm}^{2}$ have been measured by various investigators [Frye et al., 1966; Peterson et al., 1967; Duthie, 1968; Fazio et al., 1968; Fichtel et al., 1969]. These data were collected at times near solar minimum and at geomagnetic cutoff values near $4.5 \mathrm{Gv}$. They refer to photons moving approximately in the vertical direction, except for the omnidirectional measurement of Peterson et al. The spectrum of Frye et al. is adjusted to the 


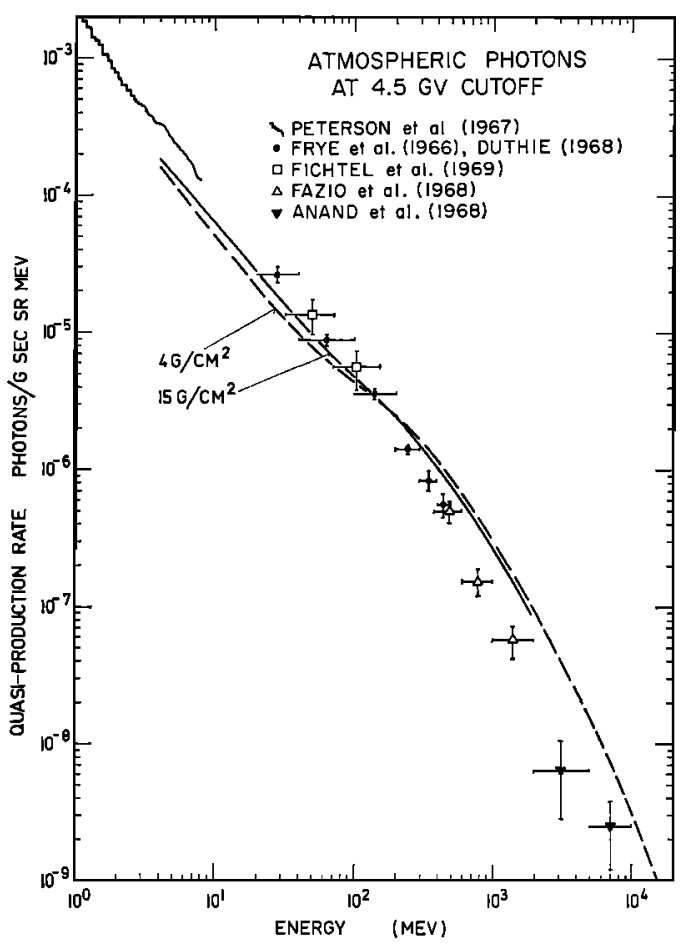

Fig. 10. Differential energy spectrum of photons at balloon altitude as measured by various investigators near solar minimum and at approximately $4.5-\mathrm{Gv}$ cutoff. The quantity plotted is the flux at a depth $x$ in $\mathrm{g} / \mathrm{cm}^{2}$ divided by $x$. Also shown are the calculated energy spectra for 1968 at 4 and $15 \mathrm{~g} / \mathrm{cm}^{2}$, divided by 4 and 15 , respectively. For the normalization of the data of Peterson et al. [1967], see text.

absolute flux reported by Duthie. The combined spectrum is shown in Figure 10, together with two data points by Anand et al. [1968b] obtained at $16.9-\mathrm{Gr}$ geomagnetic cutoff. We normalize the data to an effective atmospheric depth of $1 \mathrm{~g} / \mathrm{cm}^{2}$ by plotting the differential flux observed at depth $x$ divided by $x \mathrm{~g} / \mathrm{cm}^{2}$. In order to normalize the omnidirectional measurements of Peterson et al., we assume an isotropic and depth-independent production rate and an effective production depth of $1 / \mu$, where $\mu$ is the photon attenuation coefficient. We neglect the energy dependence of $\mu$ and use the value for $1 \mathrm{Mev}, \mu=0.06 \mathrm{~cm}^{2} / \mathrm{g}$.

We also plot in Figure 10, as solid and dashed curves, our calculated spectra for 1968 and 4.5-Gv geomagnetic cutoff at $4-\mathrm{g} / \mathrm{cm}^{2}$ and 15 $\mathrm{g} / \mathrm{cm}^{2}$ atmospheric depth, normalized by the same method as the measured fluxes. The solar cycle modulation of the atmospheric photon flux between solar minimum (measurements) and 1968 (calculated curves) can be estimated from the calculations of Perola and Scarsi [1966] and amounts to $20 \%$ near $100 \mathrm{Mev}$.

The data shown in Figure 10 form a unique spectrum of atmospheric secondary photons for times near solar minimum. Dropping smoothly from the $\mathrm{Mev}$ to the Gev region, it compares well with the principal shape of the calculated spectrum. In comparing the magnitudes of the measured and calculated fluxes at energies below $100 \mathrm{Mev}$, we have to consider (1) solar modulation, which reduces the deficiency by $\sim 20 \%$; (2) an uncertainty of $\sim 30 \%$ in the flux of reentrant albedo electrons (Figure 3); and (3) the limitations of the assumptions made in normalizing the omnidirectional measurements of Peterson et al. [1967]. The agreement between calculated and measured intensities below 100 $\mathrm{Mev}$ is then considered to be satisfactory. We conclude that the likely origin for the majority of the photons with energies between $\sim \mathbf{4}$ and $\sim 70 \mathrm{Mev}$ observed at atmospheric depths of a few $\mathrm{g} / \mathrm{cm}^{2}$ and moving vertically downward is by bremsstrahlung of reentrant albedo electrons. The background of these bremsstrahlung photons masks the peak of the $\pi^{0} \rightarrow 2 \gamma$ spectrum at $68 \mathrm{Mev}$. This conclusion is not restricted to the special case of a geomagnetic cutoff of $4.5 \mathrm{Gv}$. Our calculations for zero cutoff indicate that primary electrons also represent a strong source of bremsstrahlung photons (compare Figure 6). Thus we cannot reconcile our results with the earlier findings of a $\pi^{0}$ peak clearly set off from the electromagnetic cascade spectrum [Carlson et al., 1950; Svensson, 1958].

Figure 10 also shows that, at high energies, the actual photon flux is lower than that calculated on the basis of the production spectra of Perola and Scarsi [1966] (Figure 1). Near $10 \mathrm{Gev}$, the difference is a factor of $\sim 2.5$. This factor is inferred from a recent measurement by Stephens [1969] that confirms and improves the statistically poor data of Anand et al. [1968b] shown in Figure 10. Stephens simultaneously measures electrons and photons above $1 \mathrm{Gev}$, at an effective atmospheric depth of $11.9 \mathrm{~g} / \mathrm{cm}^{2}$ and a geomagnetic cutoff of $16.9 \mathrm{Gv}$. It should be emphasized that the production rate of highenergy $\pi \rightarrow \mu \rightarrow e$ decay electrons, as determined by Stephens, also falls below that of 
Figure 1 by a factor of 2 or 3 . The common cause of these discrepancies, for electrons and for photons, is apparently an overestimate of the progenitor pion flux by Perola and Scarsi [1966]. This conclusion is supported by the fact that the pion production spectrum as derived from the muon spectrum in the deep atmosphere [Ashton and Wolfendale, 1963; Baber et al., 1968] also agrees more closely with the results of Stephens than with those of Perola and Scarsi.

Thus the 1968 production spectra shown in Figure 1 are in need of adjustment. The comparisons with experimental data suggest that the magnitudes of these adjustments should be the same for electrons from $\pi \rightarrow \mu \rightarrow e$ decays and for photons from $\pi^{0} \rightarrow 2 \gamma$ decays. The required reduction increases from 25\% near 100 Mev to $60 \%$ near $10 \mathrm{Gev}$. The intensities of knock-on negatrons and of secondaries produced by primary electrons remain unchanged. We have investigated the influence of these revised production spectra on the results of our calculations. The absolute secondary flux of the relevant components will change according to the revised production rates (Figures 4 to 6 ). The revised fluxes are not presented, here, since only part of the calculations have been repeated. The implications as to the functional form of the intensity versus depth curves are shown in Table 2, where it can be seen that the revised production rates have no significant effect on the depth dependence of the secondary flux. We emphasize that only this functional dependence of secondaries upon depth, and not their absolute flux, is used in fitting the expected secondary flux to a measured growth curve.

\section{SumMary}

We have demonstrated that the propagation of low-energy atmospheric secondary electrons and photons moving in the vertical direction can be described within the framework of onedimensional cascade theory. Important features of our approach are:

1. We use the energy-dependent differential cross sections of the electromagnetic cascade processes and allow for energy loss by ionization. Thus the calculations can be extended down to about $4 \mathrm{Mev}$.

2. The production spectra of electrons from $\pi \rightarrow \mu \rightarrow e$ decays and photons from $\pi^{0} \rightarrow 2 \gamma$ decays are derived from the calculations of Perola and Scarsi [1966] for small atmospheric depths. In addition, we consider knock-on negatrons. These production rates, adjusted for the solar cycle change, determine the absolute magnitude of the secondary flux.

3. The attenuation of the production rates with increasing atmospheric depth is calculated as a function of energy, using a simple model of the nucleonic cascade (Appendix). This feature permits the extension of the calculation to depths in excess of $400 \mathrm{~g} / \mathrm{cm}^{2}$.

4. We consider the contribution to the secondary flux from electromagnetic cascades initiated by primary and reentrant albedo electrons.

Curves of energy spectra and intensity versus depth presented in this paper are calculated for the solar modulation level of 1968. They are compared with our own measurements of negatrons and positrons in 1968, and with experimental data of other investigators on secondary photons. Good agreement is obtained between measured and calculated energy spectra and curves of. intensity versus depth. Some differences in the magnitudes of the calculated and observed secondary fluxes are due to errors in the production spectra of Perola and Scarsi. They do not affect our other conclusions, however. Except for the absolute secondary flux, our results are valid also for periods of the solar cycle other than 1968.

We find that the spectral shapes of low-energy negatrons and positrons undergo a rapid change with increasing atmospheric depth within the first $40 \mathrm{~g} / \mathrm{cm}^{2}$ of atmosphere. This feature is not present in earlier studies neglecting energy losses [Perola and Scarsi, 1966; Verma, 1967]. The spectra reach an equilibrium state only beyond the transition maximum. The photon spectrum at energies below $\sim 70 \mathrm{Mev}$ is dominated by the cascade processes rather than by decays of neutral pions. In the high atmosphere, the peak at $68 \mathrm{Mev}$ from $\pi^{0}$ decays is masked by bremsstrahlung photons from primary and reentrant albedo electrons. This explains the steep photon spectrum observed by several investigators [e.g., Duthie, 1968].

The prime objective of this study of the propagation of atmospheric electrons and photons has been the derivation of the functional 
dependence of the secondary flux upon depth. The calculated depth dependence fits our measured growth curves over a wide depth range, from the high atmosphere to depths beyond the transition maximum. It should be emphasized that this functional depth dependence deviates considerably from the commonly assumed linear growth. Our results are therefore useful for determining the contribution of atmospheric secondaries to measurements of the electron (and photon) flux at balloon altitudes. The functional depth dependence in 1968 for depths $<40 \mathrm{~g} / \mathrm{cm}^{2}$ is given in Figure 7 . The solar cycle change of the functional depth dependence is found to be quite small (see Table 2).

\section{APPENDIX}

Here we calculate the attenuation of the pion production rate with atmospheric depth, as given in Figure 2 for high-energy pions and low-energy pions.

Nucleonic component. First, we describe a simple model of the nucleonic cascade to calculate the integral energy spectra $J_{p}(E, t)$ of protons and $J_{n}(E, t)$ of neutrons as a unique function of the atmospheric depth $t=x / \cos \theta$ along an inclined path, $x$ being the vertical depth and $\theta$ being the zenith angle. Our approach involves the stepwise integration of 2 one-dimensional transport equations, for protons and neutrons. The one-dimensional approximation applies, since the residual nucleon escapes essentially undeflected from the nuclear interaction. We assume a constant inelasticity of 0.5 , a charge exchange probability of 0.5 , and a nucleon interaction length $\lambda=80 \mathrm{~g} / \mathrm{cm}^{2}$ [Brooke et al., 1964]. We consider energy loss of protons by ionization. Allowance is also made for the production of secondary protons and neutrons from the fragmentation of $\alpha$ particles and heavier nuclei, using the work of Appa Rao et al. [1956], Jain et al. [1959], and Noon and Kaplon [1955]. The flux of the compound nuclei is attenuated exponentially with depth [McDonald, 1956; Webber and Ormes, 1967]. The interaction mean free paths for $Z>1$ particles are taken from Cleghorn et al. [1968]. The stepwise integration of the transport equations is started at the top of the atmosphere with boundary conditions given by the 1968 cosmic ray spectra of equations 2 to 4 of the main text and a vanishing neutron flux. We consider the two cases of a zero geomagnetic cutoff and a sharp cutoff at $4.5 \mathrm{Gv}$. The integral kinetic energy spectra of protons and neutrons are then calculated at intervals of $5 \mathrm{~g} / \mathrm{cm}^{2}$ for atmospheric depths $t$ up to $2000 \mathrm{~g} / \mathrm{cm}^{2}$. The flux of protons is shown to be attenuated nonexponentially. Neutrons peak near $100 \mathrm{~g} / \mathrm{cm}^{2}$. The fact that the model correctly predicts the measured proton spectrum at a depth of 700 $\mathrm{g} / \mathrm{cm}^{2}$ [Kocharian et al., 1959] lends confidence to our approach.

Angular spread of pions. The typical production angle $\varphi$ for a pion of momentum $p_{\pi}$ with respect to the trajectory of the incident nucleon is given by

$$
\langle\sin \varphi\rangle \sim\left\langle p_{\perp}\right\rangle / p_{\boldsymbol{r}}
$$

where $\left\langle p_{\perp}\right\rangle$ is the mean transverse momentum. It is commonly accepted that $\left\langle p_{1}\right\rangle \sim 0.4 \mathrm{Gev} / \mathrm{c}$, independent of energy. Consequently, pions with momenta $p_{\pi} \gtrsim 10 \mathrm{Gev} / \mathrm{c}$ continue essentially in the direction of flight of the primary particle, while pions with $p_{\pi} \sim\left\langle p_{\perp}\right\rangle$ are emitted at large angles. There is experimental and theoretical evidence that for low-energy pions a nearly isotropic spatial distribution is obtained [Camerini et al., 1952; Metropolis et al., 1958]. Hence, we calculate the depth dependence of the pion production rate for the two extreme cases: (1) for pions above $\sim 10 \mathrm{Gev} / \mathrm{c}$, using the onedimensional approximation, and (2) for pions below $\sim 0.4 \mathrm{Gev} / \mathrm{c}$ with the assumption of an isotropic production.

High-energy pions. The number of pions produced in a collision of a charge $Z$ nucleus with an air nucleus is higher by a factor $n_{\mathrm{z}}$ than the corresponding number from a nucleon-air nucleus interaction. For incident $\alpha$ particles, this mean number of interacting nucleons has been shown to be $n_{\alpha} \sim 1.8$ [Appa Rao et al., 1956; Jain et al., 1959]. On the basis of a simple overlap model for interactions induced by heavy nuclei [Noon and Kaplon, 1955], we estimate that $n_{M} \sim n_{B} \sim 2.8$ for cosmic-ray nuclei of the $M$ and $H$ charge groups. The assumption is now made that the energy distribution of the pions depends only on the energy/ nucleon of the incident nucleus. We can then express the depth dependence of the production rate of high-energy pions traveling in the vertical direction as 


$$
\begin{aligned}
& D_{x, h z}(x) \propto \frac{J_{p}\left(E^{\prime}, x\right)+J_{n}\left(E^{\prime}, x\right)}{\lambda} \\
& +\sum_{Z=2}^{28} \frac{n_{Z}}{\lambda_{Z}} J_{Z}\left(E^{\prime}, 0\right) \exp \left(-x / \Lambda_{Z}\right)
\end{aligned}
$$

where $\lambda$ and $\lambda_{z}$ are the interaction mean free paths for nucleons and charge $Z$ nuclei [Cleghorn et al., 1968], $J_{p}\left(E^{\prime}, x\right)$ and $J_{n}\left(E^{\prime}, x\right)$ are the integral kinetic energy spectra of protons and neutrons at depth $x$ as calculated above, $J_{z}\left(E^{\prime}, 0\right)$ is the kinetic energy/nucleon spectrum of charge $Z$ nuclei incident on the top of the atmosphere, and $\Lambda_{z}$ is the attenuation length for charge $Z$ nuclei. $\Lambda_{Z}$ ranges from $45 \mathrm{~g} / \mathrm{cm}^{2}$ for $\alpha$ particles [McDonald, 1956] to $10 \mathrm{~g} / \mathrm{cm}^{2}$ for iron nuclei [Webber and Ormes, 1967]. Because of the power-law character of the highenergy cosmic ray spectrum, the shape of $D_{\pi, h t}(x)$ is insensitive to the specific value assumed for the threshold energy $E^{\prime}$. For $E^{\prime}=84$ Gev/nucleon, we obtain curve 1 in Figure 2. This curve is well approximated by an exponential attenuation with $\Lambda=120 \mathrm{~g} / \mathrm{cm}^{2}$, in accordance with experimental observations on the depth dependence of high-energy interactions in the atmosphere [Perkins, 1961].

Low-energy pions. Isotropic production was assumed for pions with momenta $p_{\pi}<\left\langle p_{1}\right\rangle$, i.e., with energies below $\sim 200$ Mev. Most of these pions are produced by relatively lowenergy nuclei, which, for kinetic reasons, produce only 1 pion. In order to simplify the calculation, the assumption is made that any interacting nucleon with energy above a threshold energy $E^{\prime \prime}$ produces exactly 1 pion below $200 \mathrm{Mev}$ (independent of the production of additional pions at higher energies). The problem is thus reduced to calculating the number of collisions per gram of air as a function of atmospheric depth. It should be noted that the result will then resemble the depth dependence of the star production rate, as observed in nuclear emulsions [e.g., Lord, 1951]. Despite the simplifications, the model still retains the important spatial features of low-energy pion production.

Interactions of heavy nuclei are treated as above, assuming the production of $n_{z}$ pions per collision. Neglecting albedo nuclei, we obtain the depth dependence of the low-energy pion production rate as

$$
\begin{aligned}
D_{\pi, \text { low }}(x) \propto & \frac{1}{\lambda} \int_{0}^{2 \pi}\left[J_{p}\left(E^{\prime \prime}, x / \cos \theta\right)\right. \\
& \left.+J_{n}\left(E^{\prime \prime}, x / \cos \theta\right)\right] d \Omega \\
& +\sum_{Z=2}^{28} \frac{n_{Z}}{\lambda_{Z}} \int_{0}^{2 \pi} J_{Z}\left(E^{\prime \prime}, 0\right) \\
& \cdot \exp \left(-x / \Lambda_{Z} \cos \theta\right) d \Omega
\end{aligned}
$$

where $\theta$ is the zenith angle and $d \Omega$ is the solidangle element. The integral, being performed over the upper hemisphere, is a Gross transformation of the vertical intensity $J\left(E^{\prime \prime}, x\right)$. The functional form of $D_{\pi, \text { low }}(x)$ depends on the specific value chosen for the threshold energy $E^{\prime \prime}$. The effective threshold for pion production is estimated from the paper of Perola and Scarsi [1966] as $0.7 \mathrm{Gev} /$ nucleon. For a zero geomagnetic cutoff, we show $D_{\pi, 1 \text { ow }}(x)$ as curve 2 in Figure 2. For $4.5-\mathrm{Gv}$ geomagnetic cutoff, $D_{\pi, \text { low }}(x)$ is a flatter function of depth in the high atmosphere and closely agrees with curve 1 in Figure 2, the depth dependence of the production rate of high-energy pions.

Acknowledgments. I am grateful to Professor R. E. Vogt for his critical reading of the manuscript and for offering valuable suggestions. It is also a pleasure to thank Professor E. C. Stone for useful discussions and comments. The experimental data reported here were gathered jointly with Dr. C. J. Rice and other members of the Caltech Space Science Laboratory. I thank Dr. C. J. Rice for useful comments and for providing the least squares fit data. Mr. M. Casteel and Mr. H. E. Smith wrote the computer programs. This work was completed after my return to the Institut für Reine und Angewandte Kernphysik of the University of Kiel, W. Germany.

This work was supported by NASA grants NGL05-002-007 and NGR 05-002-160.

The Editor wishes to thank L. Peterson and W. R. Webber for their assistance in evaluating this paper.

\section{REFERENCES}

Anand, K. C., R. R. Daniel, S. A. Stephens, B. Bhowmik, C. S. Krishna, P. K. Aditya, and R. K. Puri, Rigidity spectrum of cosmic-ray helium nuclei $\geq 12 \mathrm{Gv}$, Can. J. Phys., 46, S652, 1968a.

Anand, K. C., R. R. Daniel, and S. A. Stephens, High-energy $\gamma$-rays of cosmic origin, Can. J. Phys., 46, S484, 1968 b.

Appa Rao, M. V. K., R. R. Daniel, and K. A. Neelakantan, Nuclear disintegrations produced 
in nuclear emulsions by $\alpha$-particles of great energy, Proc. Indian Acad. Sci., 43A, 181, 1956.

Ashton, F., and A. W. Wolfendale, The momentum spectrum of cosmic-ray muons at an angle of $80^{\circ}$ to the zenith at sea-level, Proc. Phys. Soc. London, 81, 593, 1963.

Baber, S. R., W. F. Nash, and B. C. Rastin, The momentum spectrum of muons at sea-level in the range 3-1000 Gev/c, Nucl. Phys., B4, 539, 1968.

Beedle, R. E., and W. R. Webber, Measurements of cosmic-ray electrons in the energy range 4 Mev to 6 bev at $2 \mathrm{~g} / \mathrm{cm}^{2}$ atmospheric depth at Ft. Churchill, Can. J. Phys., 46, S1014, 1968.

Beuermann, K. P., C. J. Rice, E. C. Stone, and R. E. Vogt, Cosmic-ray negatron and positron spectra between 12 and $220 \mathrm{Mev}$, Phys. Rev. Lett., 22, 412, $1969 a$.

Beuermann, K. P., C. J. Rice, E. C. Stone, and R. E. Vogt, Cosmic-ray negatron and positron spectra observed near Fort Churchill in 1968, Proc. 11th Intern. Cosmic Ray Conf., Budapest, paper OG 30, $1969 b$.

Bleeker, J. A. M., J. J. Burger, A. J. M Deerenberg, A. Scheepmaker, B. N. Swanenburg, and Y. Tanaka, Energy spectrum of primary electrons in the range 0.5-30 Gev, Can. J. Phys., 46, S522, 1968.

Brooke, G., P. J. Hayman, Y. Kamiya, and A. W. Wolfendale, The interrelation of the primary and sea-level spectra of cosmic rays, Proc. Phys. Soc. London, 83, 853, 1964.

Butcher, J. C., and H. Messel, Electron number distribution in electron-photon showers in air and aluminum absorbers, Nucl. Phys., 20, 15, 1960.

Camerini, U., W. O. Lock, and D. H. Perkins, The analysis of energetic nuclear encounters occurring in photographic emulsions, in Progress in Cosmic Ray Physics, vol. 1, North-Holland, Amsterdam, 1952.

Carlson, A. G., J. E. Hooper, and D. T. King, Nuclear transmutations produced by cosmicray particles of great energy, 4, The neutral mesons, Phil. Mag., 41, 701, 1950.

Chow, B. S., K. K. Wu, N. Simpson, and V. D. Hopper, Cosmic-ray flux variation with height in the atmosphere, Can. J. Phys., 46, S1020, 1968.

Cleghorn, T. F., P. S. Freier, and C. J. Waddington, The energy dependence of the fragmentation parameters and mean free paths of cosmic-ray nuclei with $Z \geq 10$, Can. J. Phys., 46, S572, 1968.

Cline, T. L., G. H. Ludwig, and F. B. McDonald, Detection of interplanetary 3- to $12-\mathrm{Mev}$ electrons, Phys. Rev. Lett., 13, 786, 1964.

Daniel, R. R., and S. A. Stephens, Recent observations on cosmic electrons and their consequences on different cosmic ray models and related astrophysical quantities, Proc. Indian Acad. Sci., 65A, 319, 1967.
Duthie, J. G., Gamma-ray astronomy, Can. J. Phys., 46, S401, 1968.

Fanselow, J. L., The primary cosmic-ray electron spectrum between 0.09 and 8.4 bev in 1965, Astrophys. J., 152, 783, 1968.

Fazio, G. G., H. F. Helmken, S. J. Cavrak, Jr., and D. R. Hearn, Search for cosmic gamma radiation with a vidicon spark chamber, Can. $J$. Phys., 46, S427, 1968.

Fichtel, C. E., D. A. Kniffen, and H. B. Ögelman, Gamma-ray astronomy balloon results, $N A S A$ GSFC preprint X-611-69-58, 1969.

Frye, G. M., F. Reines, and A. H. Armstrong, Search for solar and cosmic gamma rays, $J$. Geophys. Res., 71, 3119, 1966.

Israel, M. H., Cosmic-ray electrons between 12 Mev and $1 \mathrm{Gev}$ in 1967, J. Geophys. Res., 74, 4701, 1969.

Israel, M. H., and R. E. Vogt, Flux of cosmicray electrons between 17 and $63 \mathrm{Mev}$, Phys. Rev. Lett., 20, 1053, 1968.

Jain, P. L., E. Lohrmann, and M. Teucher, Heavy nuclei and $\alpha$-particles between 7 and $100 \mathrm{Bev} /$ nucleon, 2, Fragmentation and meson production, Phys. Rev., 115, 643, 1959.

Kocharian, N. M., G. S. Saakian, and Z. A. Kirakosian, Energy spectra and nuclear interactions of cosmic-ray particles, Soviet Phys. JETP, English Transl., 8, 933, 1959.

L'Heureux, J., The primary cosmic-ray electron spectrum near solar minimum, Astrophys. J., 148, 399, 1967.

L'Heureux, J., and P. Meyer, The primary cosmicray electron spectrum in the energy range from $300 \mathrm{Mev}$ to $4 \mathrm{bev}$ from 1964 to 1966 , Can. J. Phys., 46, S892, 1968.

Lord, J. J., The altitude and latitude variation in the rate of occurrence of nuclear disintegrations produced in the stratosphere by cosmic rays, Phys. Rev., 81, 901, 1951.

McDonald, F. B., Direct determination of primary cosmic-ray alpha-particle energy spectrum by new method, Phys. Rev., 104, 1723, 1956.

MacKeown, P. K., and A. W. Wolfendale, The interpretation of the charge ratio of cosmic-ray muons, Proc. Phys. Soc. London, 89, 553, 1966.

Mathiesen, O., C. E. Long, P. S. Freier, and C. J. Waddington, The charge distribution of the energetic very heavy nuclei in the primary cosmic radiation, Can. J. Phys., 46, S583, 1968.

Metropolis, N., R. Bivins, M. Storm, J. M. Miller, G. Friedlander, and A. Turkevich, Monte Carlo calculations on intranuclear cascades, 2, Highenergy studies and pion processes, Phys. Rev., $110,204,1958$.

Noon, J. H., and M. F. Kaplon, Interactions of heavy nuclei of the cosmic radiation, Phys. Rev., $97,769,1955$.

Okuda, $H$., and $\mathrm{Y}$. Yamamoto, Cosmic rays in the upper atmosphere, Rep. Ionosphere Space Res. Japan, 19, 322, 1965.

Ormes, J. F., and W. R. Webber, Measurements 
of the primary proton and helium spectra and their modulations using a balloon-borne Cerenkov-scintillation counter, Proc. 9th Intern. Cosmic Ray Conf., London, 1, 349, 1966.

Perkins, D. H., The main features of nuclear interactions above $100 \mathrm{Gev}$, Intern. Conf. Theoret. Aspects Very-High-Energy Phenomena, sponsored by the IUPAP, CERN 5-9, June 1961, CERN 61-22, pp. 99-124, 1961.

Perola, G. C., and L. Scarsi, Flux and energy spectrum of secondary electrons in the upper atmosphere, Nuovo Cimento, $46 \mathrm{~A}, 718,1966$.

Peterson, L. E., R. L. Jerde, and A. S. Jacobson, Balloon x-ray astronomy, AIAA J., 5, 1921, 1967.

Pinkau, K., U. Pollvogt, W. K. H. Schmidt, and R. W. Hugget, Measurement of the cosmic-ray proton and alpha-particle spectra above 10 Gev/nucl, Proc. 11th Intern. Conf. Cosmic Rays, Buclapest, paper OG 61, 1969.

Richards, J. A., and L. W. Nordheim, The energy distribution in cosmic-ray showers, Phys. Rev., 74, 1106, 1948.

Rossi, B., High Energy Particles, Prentice Hall, Englewood Cliffs, N. J., 1952.

Simnett, G. M., and F. B. McDonald, Observations of cosmic-ray electrons between 2.7 and 21.5 Mev, NASA GFSC preprint $X-611-68$ 450, 1968.

Stephens, S. A., Atmospheric electron spectrum over Hyderabad and a study of re-entrant albedo electrons (paper Mo 121) and energy spectrum of atmospheric gamma rays and its implication on the characteristics of high-energy interactions (paper Mo 122), Proc. 11th Intern. Conf. Cosmic Rays, Budapest, 1969.

Svensson, G., The cosmic-ray photon and $\pi^{0}$ meson energy spectrum at $29-30 \mathrm{~km}$ above sea level, Arkiv Fys. 13, 347, 1958.

Verma, S. D., A calculation of the flux and energy spectrum of secondary electrons at high altitudes in the atmosphere, Proc. Indian Acad. Sci., 66A, $125,1967$.

Webber, W. R., The spectrum and charge composition of the primary radiation, Handb. Phys., 46, part 2, 1967 .

Webber, W. R., A diurnal variation of the intensity and energy spectrum of low-energy electrons incident on Ft. Churchill, Canada, $J$. Geophys. Res., 73, 4905, 1968.

Webber, W. R., and C. Chotkowski, A determination of the energy spectrum of extraterrestrial electrons in the energy range of 70-2000 Mev, J. Geophys. Res., 7\%, 2783, 1967.

Webber, W. R., and J. F. Ormes, Cerenkovscintillation counter measurements of nuclei heavier than helium in the primary cosmic radiation, 1, Charge composition and energy spectra between $200 \mathrm{Mev} /$ nucleon and $5 \mathrm{bev} /$ nucleon, J. Geophys. Res., \%2, 5957, 1967.

(Received September 21, 1970; accepted March 15, 1971.) 